1 (C) 2019, American Psychological Association. This paper is not the copy of record and may

2 not exactly replicate the final, authoritative version of the article. Please do not copy or cite

3 without authors' permission. The final article will be available, upon publication, via its

$4 \quad$ DOI: $10.1037 / p s p a 0000158$

5

6

7

8

When Mere Action Versus Inaction Leads to Robust Preference Change

10

Zhang Chen

12

Radboud University

13

Rob W. Holland

14

Radboud University and University of Amsterdam

15

Julian Quandt, Ap Dijksterhuis and Harm Veling

16

Radboud University

17

18

19

20 
Author Notes

Zhang Chen, Behavioural Science Institute, Radboud University; Rob W. Holland,

23 Behavioural Science Institute, Radboud University and Faculty of Social and Behavioural

24 Sciences, University of Amsterdam; Julian Quandt, Behavioural Science Institute, Radboud

25 University; Ap Dijksterhuis, Behavioural Science Institute, Radboud University; Harm Veling,

26 Behavioural Science Institute, Radboud University.

27 We thank Linda Schmale, Roos Greven, Evert Palm, Wouter Aarts, Michelle de With and

28 Gerden Ibrahim for assistance with data collection; Fabienne Voncken, Simke van Oijen and

29 Sjors van de Schoot for creating the candy images; Xin Gao, Haokui Xu and members of the

30 Food Choice on Impulse lab for helpful comments on earlier versions of the manuscript. The

31 data of Experiments 1 and 2 have been reported in a master thesis by Linda Schmale at the

32 University of Amsterdam. The data of Experiments 5 and 6 have been reported in bachelor

33 theses by Roos Greven and Evert Palm at Radboud University. The data of Experiment 7 have

34 been reported in bachelor theses by Wouter Aarts, Michelle de With and Gerden Ibrahim at

35 Radboud University. Parts of the data reported here have been presented at the 2016 and 2017

36 annual meetings of Dutch Association of Social Psychology (ASPO; the Netherlands, 2016 and

37 2017), and the preconference on the self-regulation of health at the 18th General Meeting of the

38 European Association of Social Psychology (Spain, 2017) by the first author.

39 Zhang Chen is now at the Department of Experimental Psychology, Ghent University.

40 Correspondence concerning this article should be addressed to Zhang Chen, Department of

41 Experimental Psychology, Henri Dunantlaan 2, B-9000 Gent, Belgium. E-mail:

42 zhang.chen@ugent.be 
Abstract

46 Understanding the formation and modification of preferences is important for explaining human

47 behavior across many domains. Here we examined when and how preferences for food items can

48 be changed by linking mere action versus inaction to these items. In 7 preregistered experiments,

49 participants were trained to consistently respond to certain food items (go items) and not respond

50 to other items (no-go items) in a go/no-go training. Next, to assess preferences, they repeatedly

51 chose between go and no-go items for consumption. Decision time during the choice task was

52 manipulated and measured. Immediately after training, participants chose go items more often

53 for consumption when choosing under time pressure, for both high-value and low-value choice

54 pairs. Preferences were reliably changed in favor of go items for choices between unhealthy

55 foods, between healthy foods, and between healthy and unhealthy foods. Furthermore,

56 preference change was still observed one week after training, although the effect size largely

57 decreased. Interestingly, when participants made choices without time pressure, the effect

58 became weaker and statistically non-significant. These results suggest that preference change

59 induced by mere responding versus not responding is constrained to situations where people take

60 little time to make decisions, and the effect is relatively short-lived. By showing the reliability,

61 generalizability and boundary conditions of the effect, these findings advance our understanding

62 of the underlying mechanisms of go/no-go training, provide more insights into how the training

63 can be effectively applied, and raise new theoretical questions on how mere action versus

64 inaction impacts preferences.

65 Keywords: preference, food choice, go/no-go task, stimulus-response association, behavioral

66 change 


\section{When Mere Action Versus Inaction Leads to Robust Preference Change}

Everyday life presents numerous occasions in which we need to indicate our preferences

70 by making choices. From the mundane situations of deciding which clothes to wear and what

71 foods to eat, to more important decisions such as which job to take and where to live, preferences

72 are expressed in all the choices that we make in various life domains. It is therefore important to

73 understand how preferences are formed and may be modified.

74 While some preferences are innate (e.g., preferences for sugary, salty and fatty foods;

75 Breslin, 2013), we humans can also acquire new preferences and modify existing ones (including

76 some innate preferences) by learning from experiences. One prominent form of learning is

77 reinforcement learning, in which different courses of action lead to either reward or punishment

78 (Sutton \& Barto, 1998). After an organism learns the contingencies between its responses and the

79 rewarding or punishing outcomes, responses that lead to reward become preferred and are more

80 likely to occur again than responses that lead to punishment (the law of effect; Thorndike, 1911).

81 The principles of reinforcement learning play an important role in the creation and modification

82 of preferences, and have been implicated in many recent models of decision-making (e.g., Dayan

83 \& Niv, 2008; Doya, 2008; Rangel, Camerer, \& Montague, 2008).

84 Interestingly, some recent work suggests that simple responses that are not reinforced by

85 reward or punishment may also lead to preference change (Schonberg et al., 2014). For instance,

86 consistently responding to certain objects (e.g., pressing a key on a keyboard; go items) and

87 withholding responses toward other objects (no-go items) have been shown to create preferences

88 for go items over no-go items under some conditions (Bakkour et al., 2016; Schonberg et al.,

89 2014; Veling, Chen, et al., 2017; Zoltak, Veling, Chen, \& Holland, 2018). Notably, no reward or

90 punishment was delivered after the go/no-go responses in these experiments, suggesting that 
91 manipulating non-reinforced go/no-go responses toward objects can change people's preferences.

92 However, it is unclear whether mere action versus inaction per se, or other processes that

93 accompany the execution or restraint of responses (e.g., attentional process), lead to the

94 preference change effect. Understanding the role of mere action versus inaction in the

95 modification of preferences is important, as human behaviors, despite their apparent diversity,

96 can be reduced into two fundamental categories: the execution of behavioral responses, or the

97 absence thereof (Guitart-Masip, Duzel, Dolan, \& Dayan, 2014). In the current research, we are

98 hence interested in when and how linking mere action versus inaction to objects in the absence of

99 other potentially confounding factors can influence people's preferences for these objects.

100 Different tasks have been used to manipulate responding versus not responding to objects,

101 such as the stop-signal training (SST; Lawrence, Verbruggen, Morrison, Adams, \& Chambers,

102 2015; Wessel, Doherty, Berkebile, Linderman, \& Aron, 2014), the cue-approach training (CAT;

103 Schonberg et al., 2014) and the go/no-go training (GNG; Veling, Holland, \& van Knippenberg,

104 2008). In all three trainings, participants are presented with images of different objects and asked

105 to execute or withhold a simple response (e.g., press a key on a keyboard) depending on a cue (or

106 the absence of cue) that is presented in close temporal proximity with the image. The pairing

107 between an item and a response cue is often consistent, so that for some items participants

108 always respond, while for other items they always do not respond during the training.

109 Importantly, some procedural differences exist between the trainings. In SST, no-go trials

110 are accompanied by a no-go cue, while no cue is presented on go trials. The training contains

111 more go trials than no-go trials. The reverse is true for CAT: only go cues are used, and the

112 training contains more no-go trials than go trials. Because of these differences between the go

113 and no-go trials in SST and CAT, effects of SST and CAT on preferences may not be completely 
114 explained by mere action versus inaction. For instance, effects of CAT on preferences have been

115 explained by sustained attention rather than by mere motor responses (Bakkour et al., 2016). In

116 contrast, the go and no-go trials in GNG are closely matched. Both go trials and no-go trials in

117 GNG are accompanied by a cue that signals to respond or not respond, respectively. Furthermore,

118 GNG generally includes an equal number of go and no-go trials. We therefore used GNG in the

119 current research to assess whether mere action versus inaction can impact preferences.

120 Although manipulating go/no-go responses toward objects with GNG has been shown to

121 change people's preferences (Porter et al., 2018; Veling, Aarts, \& Stroebe, 2013a, 2013b), the

122 mechanisms underlying the preference change effect have remained unclear. In the current

123 research, we investigated three important theoretical questions concerning the effect of GNG on

124 preferences, namely (1) whether the effect would be influenced by the amount of decision time

125 used to indicate one's preference; (2) the durability of the preference change effect; and (3)

126 whether the initial reward value of objects would moderate the effect. As explained below,

127 answering these three questions provides insights into when merely responding versus not

128 responding to objects can lead to preference change, and advances knowledge on the underlying

129 mechanisms that give rise to this effect.

Decision Speed

131 Decision speed, or information processing speed in general, features prominently in

132 decades of theorizing on judgment and decision-making (Kahneman, 2011). Based on processing

133 speed, some models have dichotomized mental processes into one of two distinct types, one that

134 is fast and impulsive, and one that is slow and reflective (e.g., the reflective-impulsive model;

135 Strack \& Deutsch, 2004). Other models have treated processing speed as a continuum where

136 more time allows for the integration of more information into a decision process (e.g., Berkman, 
137 Hutcherson, Livingston, Kahn, \& Inzlicht, 2017; Forstmann, Ratcliff, \& Wagenmakers, 2016;

138 Krajbich, Armel, \& Rangel, 2010). Both types of models converge on the idea that decisions

139 differ as a function of decision speed, with fast decisions based on more basic information that is

140 readily accessible. For instance, when making food choices, fast choices tend to be more strongly

141 based on basic features such as tastefulness rather than more complex features such as

142 healthfulness (Friese, Hofmann, \& Wänke, 2008; Sullivan, Hutcherson, Harris, \& Rangel, 2015).

143 GNG has been proposed as a useful behavior change intervention, because it may change

144 behavior under conditions where people do not take much time to think about their responses.

145 Specifically, the reflective-impulsive model (Strack \& Deutsch, 2004) has been used as a

146 theoretical framework to explain the effects of GNG (e.g., Veling, Aarts, \& Papies, 2011; Veling,

147 van Koningsbruggen, Aarts, \& Stroebe, 2014). According to the reflective-impulsive model,

148 repeatedly executing certain responses toward objects in people's daily lives (e.g., approach and

149 consume palatable foods) may lead to the acquisition of associative links between the objects and

150 responses. Once acquired, the associative links can strongly impact people's behavior when time

151 is limited and people do not carefully reflect on their behaviors (e.g., the mere perception of

152 palatable foods may trigger approach tendency; Seibt, Häfner, \& Deutsch, 2007). To change

153 people's behavior then, GNG can be used to modify these learned associative links, so that

154 behavior is changed even when people do not carefully reflect on their behaviors. However,

155 evidence for this claim is lacking, as to date no study compared the effects of GNG between

156 situations where people can think about what to do and situations where this opportunity for

157 reflection is reduced.

158 To complicate matters, some findings seem to suggest the opposite, that GNG influences

159 behavior when there is sufficient time to think about one's responses. Specifically, responding or 
160 not responding to objects has been shown to reliably change evaluations of these objects (i.e., no-

161 go items are evaluated less positively than go or untrained items), when evaluation was assessed

162 with explicit self-reports (Chen, Veling, Dijksterhuis, \& Holland, 2016, 2018a; Doallo et al.,

163 2012; Frischen, Ferrey, Burt, Pistchik, \& Fenske, 2012; Lawrence, Sullivan, et al., 2015; Veling

164 et al., 2008). In these studies, participants had unlimited time to indicate their evaluations. In

165 contrast, a meta-analysis indicates that when evaluations are assessed with the implicit

166 association test, a reaction time measure that encourages speeded responses (Greenwald,

167 McGhee, \& Schwartz, 1998), no effects of GNG on evaluation are found (Jones et al., 2016).

168 These results thus seem to suggest that GNG changes behavior in situations where there is

169 enough time to reflect on one's responses.

170 It is thus unclear whether preference change induced by GNG would be weaker or

171 stronger depending on the amount of decision time available for making choices. GNG may

172 modify associations with objects (e.g., associations between objects and basic affective or motor

173 responses), which may be quickly incorporated into a decision process (Berkman et al., 2017;

174 Strack \& Deutsch, 2004). In that case, effects of GNG may be more visible when people have

175 little time to indicate their preferences. Alternatively, GNG may modify one's explicit

176 knowledge about go or no-go items, such as explicit evaluations or knowledge about the

177 contingencies between specific items and responses, which may require more time to be

178 incorporated into a decision. This would mean that the effects of GNG might be stronger when

179 there is more time for making choices. To gain insight into this question, we examined whether

180 the effect of GNG on preferences depends on how much time people have for making choices,

181 and for this we manipulated the amount of decision time available in the choice task. Since 
182 decision time varies on a continuum, we also conducted an exploratory analysis, in which

183 decision time was used as a continuous predictor of choice.

\section{The Durability of Preference Change}

185

The second important question concerns the durability of the effects induced by GNG. A

186 recent meta-analysis on the effects of GNG and SST on health behavior showed that when

187 behavior was assessed one to seven days after training, the overall effect size was already smaller

188 than immediately after training (Allom, Mullan, \& Hagger, 2016). However, since different

189 measures were often used at different time points, it is unclear whether this decrease in effect

190 size truly reflects the decay of the training effects, or is due to the different measurements

191 employed to assess behavior. To overcome this problem, and to have an objective measure of the

192 effect size of the potential decay, we measured preference both immediately after training and

193 one week later in the current research, with the same behavioral measurement.

194 Note that the seemingly short-term influence of GNG on behavior stands in stark contrast

195 to preference change induced by CAT, which has been shown to be highly durable in retest

196 sessions performed one to six months after training (Salomon et al., 2018; Schonberg et al.,

197 2014). Strikingly, in a six-month follow-up study in Salomon et al., (2018), participants still

198 preferred go items around $60 \%$ of the time, showing a decrease of merely 4 percentage points

199 compared to immediately after training. One explanation for this potential difference in the

200 longevity of training effects by GNG and CAT are the different processes via which the trainings

201 influence behavior. Another explanation may be the different dependent measurements used in

202 these two lines of research. Till now, almost all studies with CAT have used choice as the

203 dependent measurement (Bakkour et al., 2016; Salomon et al., 2018; Schonberg et al., 2014;

204 Veling, Chen, et al., 2017; Zoltak et al., 2018), whereas studies with GNG have employed a wide 
205 range of behavioral measures, with choice occasionally measured as the outcome of training

206 (Porter et al., 2018; Veling et al., 2013b, 2013a). This methodological difference makes the

207 comparison between GNG and CAT difficult. In the current research, we focused on the effect of

208 GNG on choices, and used the same choice task that has been used in previous work on CAT.

209 Our aim was to provide a high-quality dataset on the effect of GNG on choices, which can then

210 allow a comparison between the results obtained by CAT and GNG. Such a comparison will

211 provide insights into which task works better to induce long-term preference change.

The Role of Reward Value

213 The third question concerns whether the effect of GNG on preferences would be

214 moderated by the reward value of objects. This question is interesting as it potentially pits two

215 accounts that explain the effects of GNG against each other (for a recent theoretical review, see

216 Veling, Lawrence, Chen, van Koningsbruggen, \& Holland, 2017). According to the first account,

217 the Behavior Stimulus Interaction theory (BSI theory, Veling et al., 2008), appetitive items

218 automatically trigger approach tendencies that need to be inhibited when a no-go cue is presented.

219 The response conflict resulting from approach tendency and response inhibition is negative

220 (Dreisbach \& Fischer, 2015; Fritz \& Dreisbach, 2013). The negativity is then attached to no-go

221 items through repeated pairings, thereby decreasing the reward value of (Chen et al., 2016;

222 Veling et al., 2008) and the preference for no-go items.

223 Repeatedly not responding to an object can also create stimulus-stop associations (Best et

224 al., 2016; Verbruggen, Best, Bowditch, Stevens, \& McLaren, 2014; Verbruggen \& Logan, 2008).

225 Once formed, response inhibition can be automatically triggered and interferes with responses

226 toward no-go items, for instance reducing the frequency or vigor of responses. Since preference 
227 is often expressed with certain responses (e.g., pointing at or grabbing a preferred object),

228 inhibiting responses toward no-go items may hence reduce preferences for no-go items.

229 These two accounts make different predictions for whether GNG will lead to preference

230 change for low-value items. According to the BSI theory, no-go response changes preferences

231 only when the item possesses high reward value, as response conflict only arises when people

232 have strong initial approach tendencies (Chen et al., 2016; Veling et al., 2008). The stimulus-stop

233 association account, on the other hand, does not assume a causal role of response conflict and

234 predicts preference change for both high-value and low-value items. Most previous work till now

235 has used positive items in the training, and focused on evaluation rather than preference. In the

236 few cases where the effect of GNG on evaluation of neutral or negative items was examined,

237 results were mixed (Chen et al., 2016; Chen, Veling, Dijksterhuis, et al., 2018a; Frischen et al.,

238 2012; Veling et al., 2008). Systematically examining whether GNG changes preference for low-

239 value items, in addition to high-value items, will therefore be theoretically informative in

240 distinguishing the two accounts.

The Present Research

242 To address the three questions outlined above, we conducted 7 experiments. In all

243 experiments, participants were trained to consistently respond to certain food items (both of high

244 value and of low value; go items) and withhold their responses toward other food items (no-go

245 items) in GNG. After the training, they repeatedly chose between go and no-go items in a food

246 choice task for consumption, with either limited (1.5 seconds for each choice; Experiments 2, 4-7;

247 see Schonberg et al., 2014; Veling, Chen, et al., 2017) or unlimited time (Experiments 1 and 3).

248 The durability of preference change was explored by measuring preferences both immediately

249 following training and one week later (Experiments 4-6). For a summary of the key design 
250 features across all experiments, see Table 1. Food choice was selected as the main dependent

251 variable, to enable comparisons with previous work (Schonberg et al., 2014; Veling et al.,

252 2013b). A second reason for targeting food choice is that many people nowadays are struggling

253 with making healthy food choices. With the rising rate of overweight and obesity worldwide

254 (World Health Organisation, 2016), our ability to address the obesity epidemic depends on our

255 understanding of how food choices may be changed.

256 For the sake of transparency, we preregistered all 7 experiments. Preregistrations

257 containing planned sample sizes, analysis plans and directional hypotheses can be found at

258 https://osf.io/zy9w3/. Experimental materials (stimuli and Python scripts), raw data and analyses

259 scripts are also available. For deviations from preregistrations and the reasons for deviations, see

260 Footnotes 1 and 2.

261 At the start of the project, we predicted preference change for high-value items when

262 people have unlimited time. This prediction was based on the findings that GNG reliably

263 devalued high-value items when evaluation was measured with explicit self-report without time

264 pressure (Chen et al., 2016; Veling et al., 2008). To preview the results, we found that GNG led

265 to preference change for both high-value and low-value items when decision time was limited,

266 but not when people chose with unlimited time. Preference change was still observed one week

267 after training, but the effect size was smaller than immediately after training. Our theoretical

268 stance was adjusted based on the observed results during the project. Specific predictions can be

269 found in the introduction for each experiment and in the preregistrations.

Experiment 1 - Slow Choice

271 In Experiment 1, we investigated the effect of GNG on preference when people had

272 unlimited decision time. As mentioned above, a priori we expected GNG to lead to preference 
273 change for high-value items. For low-value items, we expected no effect. We also measured the

274 value of food items after training with an auction task, in which no time limit was implemented.

275 In line with previous work of GNG on evaluation (Chen et al., 2016; Chen, Veling, Dijksterhuis,

276 et al., 2018a; Veling et al., 2008), we expected reduction in the reward value of no-go items

277 compared to go items, but again only for high-value items ${ }^{1}$.

278 Method

279 Sample Size. Previous work on the effect of GNG on food evaluation estimated the 280 effect size of devaluation to be around Cohen's $d_{z}=0.537$ (Chen et al., 2016). Assuming the 281 effect of GNG on preference is similar to that on evaluation, we used this estimate as the 282 expected effect size. Power analysis with G*Power 3 (Faul, Erdfelder, Lang, \& Buchner, 2007)

283 suggested 30 participants for achieving $80 \%$ power (alpha level of 0.05 , paired-samples $t$ test).

284 We therefore planned to recruit 30 participants for Experiment 1. In Experiments 1 and 2, mostly

285 undergraduate students at the University of Amsterdam were recruited as participants. In

286 Experiments 3-7, mostly undergraduate students at Radboud University were recruited. Repeated

287 participation was not allowed, to ensure that each experiment would contain different samples.

288 The ethics committee at the department of psychology at the University of Amsterdam and the

289 ethics committee at the Faculty of Social Sciences at Radboud University provided ethical

290 approvals. All participants provided written informed consent before participating in the

291 experiments.

292 Participants. Thirty-one participants ${ }^{2}$ took part in Experiment 1. Based on the

293 preregistered exclusion criteria (1. participants who were not between 18 and 26 years old; 2.

294 participants who bid less than 25 cents on more than 40 food items in the first auction task; 3.

295 participants whose accuracy on go or no-go trials in the training was $3 S D$ below sample mean 
and below 90\%), 7 participants were excluded, leaving 24 participants in the final sample. For an overview of exclusion based on each criterion for all experiments, see Table $\mathrm{S} 1$ in the

298 Supplemental Materials.

Materials. Sixty images of high-calorie snacks (e.g., chips, cookies, chocolate bars, candies etc.) were selected from previous work (Veling, Chen, et al., 2017). On each image, both

301 the packaging and content were clearly visible against black background. All snacks were 302 available in local supermarkets and familiar to participants. The tasks were programmed in 303 PsychoPy (Peirce, 2007) and executed individually for each participant.

\section{Procedure.}

Preparation. Participants were asked to not eat anything for at least $3 \mathrm{hr}$ before coming

306 into the lab (drinking water was allowed). Upon arrival, they were asked to verbally report the

307 last time of food consumption to the experimenter. Those who did not adhere to the fasting

308 requirement were asked to reschedule the experiment.

Pre-Training Auction. Participants first received 2 euros (1 euro, 50 cent, 20 cent, $2 * 10$

310 cent, and $2 * 5$ cent) from the experimenter to bid in an auction task based on the Marschak-

311 DeGroot-Becker procedure (Marschak, DeGroot, \& Becker, 1964), which has been used to

312 assess the willingness to pay (WTP) for snacks in previous work (Schonberg et al., 2014; Veling,

313 Chen, et al., 2017). For each of the 60 snacks, they were asked to place a bid by moving a mouse

314 cursor along an analog scale that ranged from 0 to 2 euro (see Panel A of Figure 1). They were

315 told that at the end of the experiment, the program would randomly pick one snack and generate

316 a bid for the selected snack. If their bid was higher than the bid from the computer, they could

317 buy the snack at the computer's bid. To reduce the number of snacks we needed to purchase, we 
318 had a selection of snacks in the lab and the program picked one snack out of this reduced

319 selection. For a more detailed description of the auction rules, see the OSF repository.

320 Item Selection. After participants placed bids for all 60 snacks, the program rank ordered

321 the snacks from the highest WTP to the lowest. Eight relatively high-value items (ranked from

322 eight to 15 ) and 8 relatively low-value items (ranked from 46 to 53) were selected for each

323 participant and divided into the go and no-go condition in a counterbalanced manner. This

324 division procedure ensured that the average WTP of go and no-go items was matched before

325 training, for high-value and low-value items separately. Another 8 items (4 relatively high-value

326 and 4 relatively low-value) were further selected into the no-go condition and used on the filler

327 trials in the choice task (see Food Choice below). In total, 16 snacks were assigned to the no-go

328 condition, and the remaining 44 to the go condition. For the selection procedure, see Figure S2 in

329 the Supplemental Materials.

330 Go/No-Go Training. Participants then received the GNG (Panel B of Figure 1). In the

331 training, one snack image was presented on each trial. One hundred milliseconds after image

332 onset, a beep was played via headphone. Two different beeps were used as the go and no-go cue,

333 respectively (frequencies $1000 \mathrm{~Hz}$ and $400 \mathrm{~Hz}$, duration $300 \mathrm{~ms}$; the assignment of beeps as go

334 and no-go cues was counterbalanced across participants). If the played beep was a go cue,

335 participants needed to press the B key on the keyboard as fast as possible; if it was a no-go cue,

336 they should not respond. In both conditions, the images stayed on screen for one second to

337 control for exposure time. The inter-trial interval randomly varied between 1.5 and 2.5 seconds,

338 in steps of $100 \mathrm{~ms}$. Participants first received a practice block of 8 trials, during which an

339 incorrect response was followed by an error message ( $\mathrm{X}$ in red) for $500 \mathrm{~ms}$. The images used in

340 the practice block were not used in the experimental blocks. In the experimental blocks, no 
341 performance feedback was provided after each trial, but the overall accuracy was provided after

342 every two blocks. Each image was randomly presented once in each experimental block, and the

343 whole training consisted of 8 blocks, resulting in 480 trials in total.

344 Food Choice. Participants then received a food choice task (Panel C of Figure 1). On

345 each trial, two snacks were presented side by side, and participants chose by pressing the U (left)

346 or the I (right) key. They were told that at the end of the experiment, one trial would be randomly

347 selected and they would receive the snack chosen on the selected trial. Two rigged trials were

348 added to the end of the choice task, with snacks that were available in the lab, so that we did not

349 need to purchase all 60 snacks. The choice task consisted of two types of trials, the experimental

350 trials and the filler trials. On the experimental trials, the two snacks had matched value (both

351 high or both low, 32 unique pairs in total); one was paired with go responses in the training,

352 whereas the other was paired with no-go responses. On the filler trials, both snacks were paired

353 with go or no-go responses; one had high value, whereas the other had low value (32 unique

354 pairs in total). Different items were used on the experimental trials and the filler trials. Each pair

355 was presented twice to counterbalance the left-right position. The whole task consisted of 128

356 trials (excluding the two rigged trials). Participants received unlimited time for each choice.

357 After participants chose, the chosen item was surrounded by a yellow frame for $500 \mathrm{~ms}$ as

358 confirmation. The inter-trial interval varied between 1.0 and 2.0 seconds, in steps of $100 \mathrm{~ms}$.

359 Memory Recognition. A memory recognition task was included to assess participants'

360 memory of the snack-response contingencies. For each snack, they indicated whether it had been

361 paired with pressing B (i.e., go response) or not (i.e., no-go response) during GNG.

362 Post-Training Auction. To measure changes in food value, participants then received a

363 second auction task, with the same auction rules as the one they received before the training. 
Demographics. Finally, participants filled out demographic information such as height,

365 weight, whether they were currently on a diet, current hunger level $(-100=$ Not hungry at all;

$366100=$ Very hungry), number of hours since last food consumption, and the restraint eating scale

367 (van Strien, Peter Herman, Engels, Larsen, \& van Leeuwe, 2007). Age and gender were reported

368 when the experimental program was started. After answering the demographic questions, all

369 participants received one snack based on a trial selected from the choice task (i.e., one of the two

370 rigged choice trials). If they won the auction, they received a second snack. Participants were

371 then debriefed, compensated and thanked.

372 

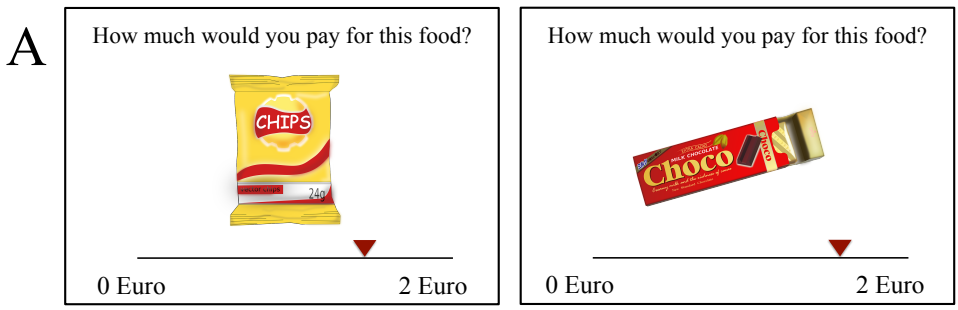

$\mathrm{B}$

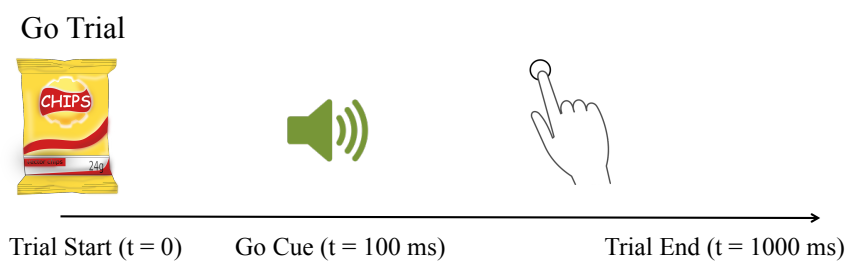

No-Go Trial

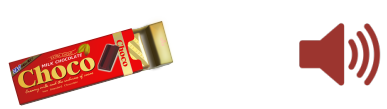

Trial Start $(\mathrm{t}=0) \quad$ No-Go Cue $(\mathrm{t}=100 \mathrm{~ms}) \quad$ Trial End $(\mathrm{t}=1000 \mathrm{~ms})$
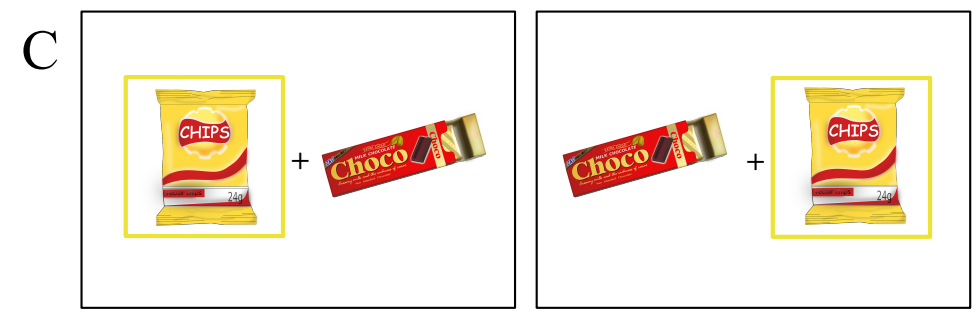

Figure 1. Sequence of Main Experimental Tasks. (A) Auction task in experiment 1, 2 and 7;

375 rating task in experiments 3-6; (B) The go/no-go training; (C) Binary choice task, with

376 (experiments 2 and 4-7) or without (experiment 1 and 3) time limit. In experiments 4-6, binary

377 choice task (C) was repeated in retest session. Images are for illustration. For the stimuli and the

378 scripts used in the experiments, see the OSF repository. 


\section{Results}

Main analyses (repeated-measures logistic regression and multilevel models) were

383 conducted with the lme4 package in R (Bates, Maechler, Bolker, \& Walker, 2015; R Core Team, 384 2017). For a summary of participant demographics (Table S2), performance in GNG (Table S3), 385 and performance in the memory recognition task (Table S4), see the corresponding tables in the

386 Supplemental Materials. An exploratory analysis on the development of GNG performance 387 across blocks is also reported in the Supplemental Materials.

388 Food Value. To check the selection procedure, the average WTP of the items selected for 389 the experimental choice trials ( 8 high-value and 8 low-value items, divided into go and no-go 390 condition) was calculated for each participant, and then submitted to repeated-measures ANOVA 391 with training condition (go vs. no-go) and value level (high vs. low) as independent variables. As

392 can be seen from Table 2, participants were willing to pay more for high-value items than for

393 low-value items. More importantly, the main effect of training condition was not significant, 394 indicating that the selection procedure succeeded in selecting go and no-go items with matched 395 WTP before training.

To see whether the training influenced the value of snacks, a repeated-measures ANOVA

397 was conducted, with measurement time (before vs. after training), value level (high vs. low) and

398 training condition of items (go vs. no-go) as the independent variables, and the average WTP

399 before and after training as the dependent variable. The interaction effect between measurement

400 time and training condition of items was close to, but did not reach statistical significance, $F(1$,

$40123)=3.92, p=.060, \eta^{2}=.002$. Before the training, there was no significant difference in WTP

402 between go and no-go items; after the training, participants were willing to pay more for the go

403 items $\left(M_{\text {high }}=1.083, S D=0.43 ; M_{\text {low }}=0.437, S D=0.35\right)$ than for the no-go items $\left(M_{\text {high }}=\right.$ 
$4040.982, S D=0.48 ; M_{\text {low }}=0.429, S D=0.36$ ), although this main effect of training condition on

405 post-training WTP was not statistically significant, $F(1,23)=3.64, p=.069, \eta^{2}=.007$.

406 Although after training, the value difference between go and no-go items was numerically larger

407 for high-value items $\left(M_{\text {diff }}=0.101\right)$ than for low-value items $\left(M_{\text {diff }}=0.008\right)$, the interaction effect

408 between value level and training condition on post-training WTP, however, was not statistically

409 significant, $F(1,23)=1.82, p=.190, \eta^{2}=.005$.

410 Food Choices. Choices from the experimental trials, where go items were paired with

411 similarly valued no-go items, were analyzed with repeated-measures logistic regression ${ }^{3}$.

412 Overall, participants did not choose go items more often than chance level, mean proportion of

413 go choices $=53.0 \%$, Odds Ratio $(\mathrm{OR})=1.14,95 \% \mathrm{CI}=[0.87,1.50], p=.327($ see Figure 2 ,

414 upper panel). Contrary to our expectation, for both the high-value and low-value choice pairs,

415 participants did not choose go items significantly more often than no-go items, mean proportion

416 of go choices $=55.1 \%, \mathrm{OR}=1.26,95 \% \mathrm{CI}=[0.90,1.78], p=.183$ and mean proportion $=$

$41750.9 \%, \mathrm{OR}=1.06,95 \% \mathrm{CI}=[0.56,2.00], p=.869$, respectively. Although numerically

418 participants did choose go items more often for the high-value pairs than for the low-value pairs,

419 the difference was not statistically significant, $\mathrm{OR}=1.19,95 \% \mathrm{CI}=[0.55,2.59], p=.653$

420 (Figure 2, lower panel).

421 On the filler trials, participants chose between high-value and low-value items, where

422 both items were paired with go or no-go responses in the training. Repeated-measures logistic

423 regression showed that participants chose high-value items more often than chance level, mean

424 proportion of choosing high-value items $=83.7 \%, \mathrm{OR}=9.69,95 \% \mathrm{CI}=[5.06,18.57], p<.001$

425 (see Figure 3). For a summary of the choices on the experimental and filler trials, as well as the

426 median choice reaction times in each experiment, see Table 3. For brevity we will not discuss the 
427 result on decision time per experiment, but will instead analyze the data from all experiments

428 combined in the end.

429 Discussion

430 Contrary to our predictions, participants did not choose go items more often when they

431 had unlimited decision time. Furthermore, the proportion of go choices did not differ

432 significantly between high-value and low-value pairs. This absence of effect on the experimental

433 trials cannot be explained by participants' indifference or lack of motivation, as they showed a

434 strong preference for high-value items on the filler trials that were intermixed with the

435 experimental trials. Results on post-training value were in the expected direction, but did not

436 reach statistical significance.

437 Due to the large number of excluded participants, the final sample consisted of 24

438 participants. The experiment may therefore have been underpowered. This potential problem of

439 insufficient power was addressed in later experiments (Experiments 3-7) by doubling the sample

440 size. Next, we continue with the investigation on the role of decision time and report the effect of

441 GNG on preference when people made choices with time limit in Experiment 2. 

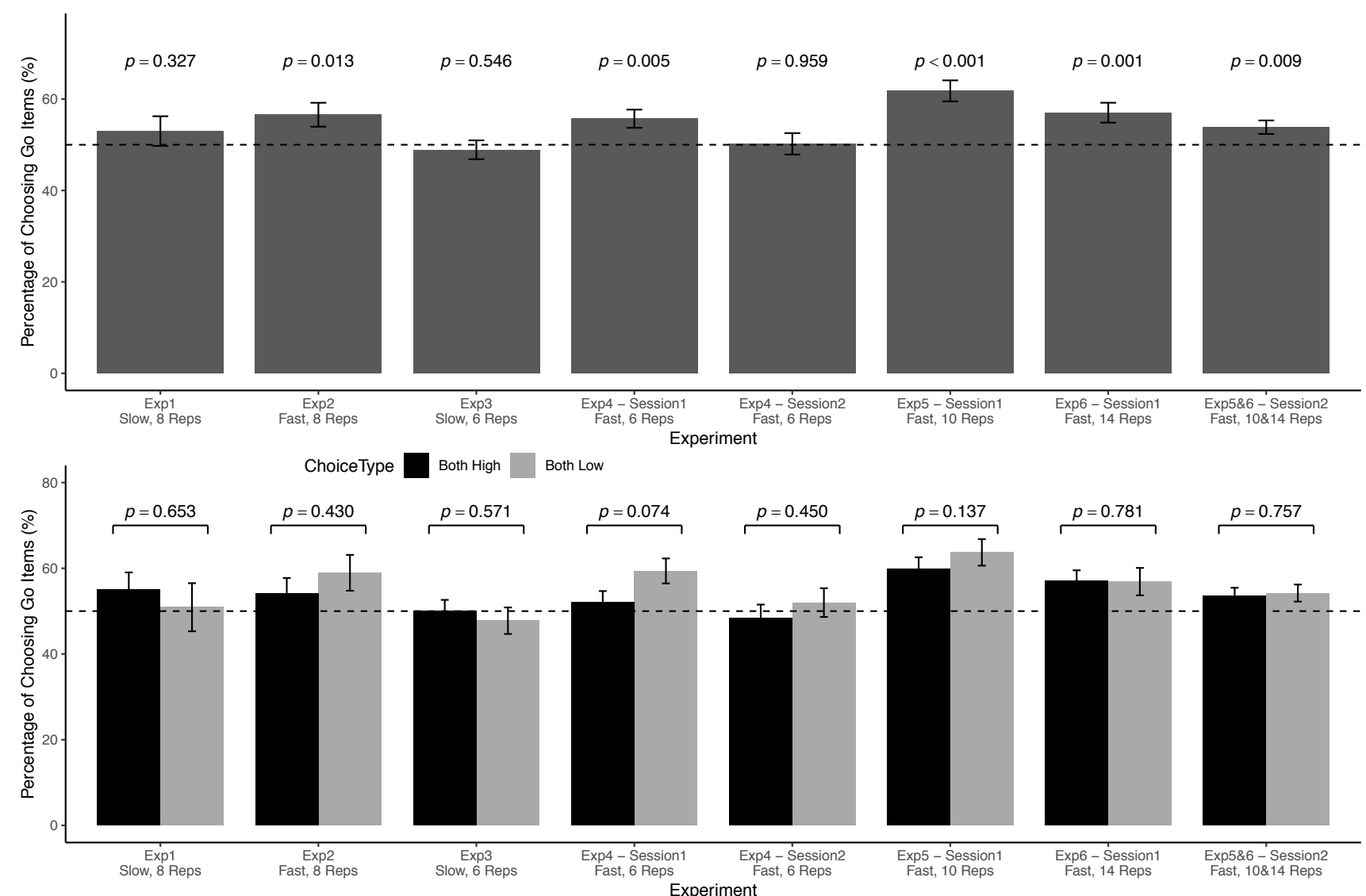

444 Figure 2. Probability of choosing go items on experimental trials in Experiments 1-6, combining

445 (upper panel) and separating (lower panel) high-value and low-value choice pairs. Slow

446 indicates choices made without time limit and fast indicates choices made within 1500

447 milliseconds. Reps indicates how many times the go and no-go items were presented in the

448 training. The $p$ values in the upper panel are calculated from repeated-measures logistic

449 regression comparing the overall probability of choosing go items against the $50 \%$ chance level.

450 The $p$ values in the lower panel are calculated from repeated-measures logistic regression

451 comparing the probability of choosing go items between high-value and low-value pairs. Error

452 bars stand for standard errors of mean proportions. 


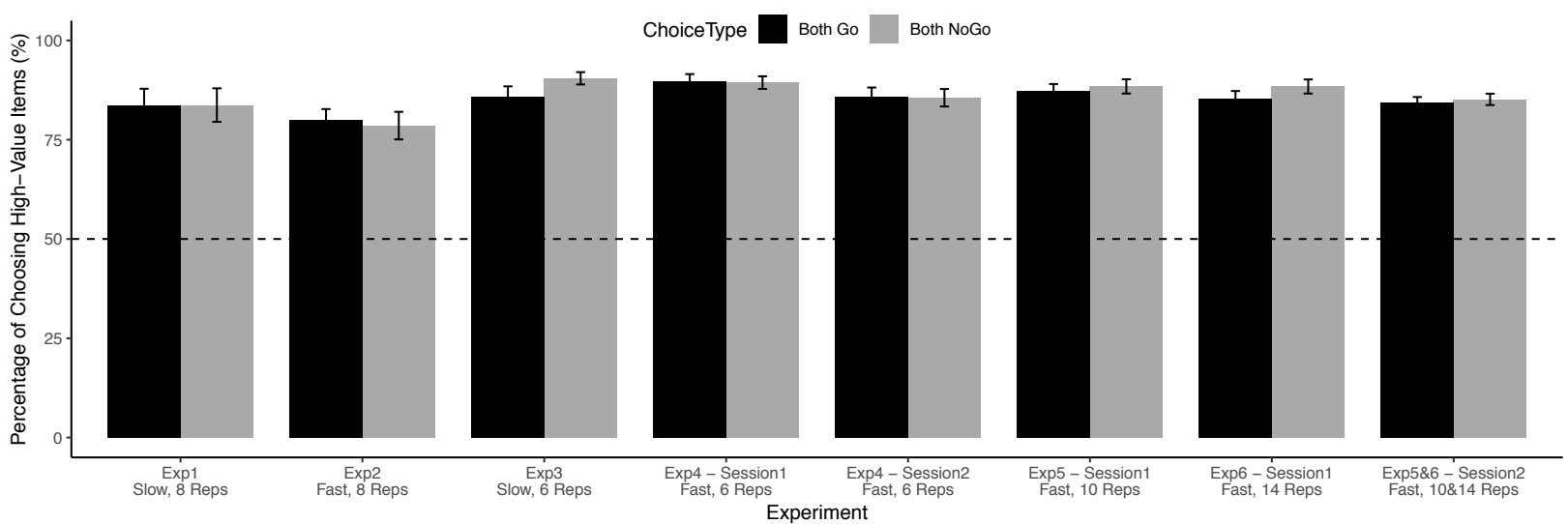

454 Figure 3. Probability of choosing high-value items on filler trials in Experiments 1-6, separated

455 for both go and both no-go pairs. Slow indicates choices made without time limit and fast

456 indicates choices made within 1500 milliseconds. Reps indicates how many times the go and no-

457 go items were presented in the training. Error bars stand for standard errors of mean proportions.

In Experiment 2, participants made choices under time limit (1.5 seconds for each choice)

460 after training. A priori we did not have directional hypothesis for whether GNG would influence

461 such fast choices. For the post-training auction task with no time limit, similar to Experiment 1,

462 we expected a devaluation effect for high-value no-go items, but not for low-value no-go items.

\section{Method}

464 Participants. Thirty-one participants participated in Experiment 2. Based on the

465 preregistered exclusion criteria (1. participants who were not between 18 and 26 years old; 2.

466 participants who bid less than 25 cents on more than 40 food items in the first auction task; 3.

467 participants whose accuracy on go or no-go trials in GNG was $3 S D$ below sample mean and

468 below 90\%), 2 participants were excluded, leaving 29 participants in the final sample.

469 Procedure. The procedure of Experiment 2 was identical to Experiment 1, except that in

470 the choice task participants had 1.5 seconds for each choice. If they did not choose in time, the 
471 current pair of snacks would be replaced by a prompt saying 'Choose Faster!' for 500 ms. The

472 missed trial would be presented again at a later point until they chose in time. If participants

473 chose in time, the chosen snack was surrounded by a yellow frame for $500 \mathrm{~ms}$ as confirmation. 
ACTION VERSUS INACTION ON PREFERENCE

475 Table 1. Summary of Experimental Designs

476

\begin{tabular}{llllll}
\hline Experiment & Stimulus & Go/No-Go Proportion & Training Length & Decision Speed & Delay (Range) \\
\hline Exp1 & Snacks & $75 \% / 25 \%$ & 8 & Slow & - \\
Exp2 & Snacks & $75 \% / 25 \%$ & 8 & Fast & Slow \\
Exp3 & Candies & $50 \% / 50 \%$ & 6 & Fast & $12.4(7-19)$ \\
Exp4 & Candies & $50 \% / 50 \%$ & 6 & Fast & $8.1(7-14)$ \\
Exp5 & Candies & $50 \% / 50 \%$ & 10 & Fast & $8.3(7-14)$ \\
Exp6 & Candies & $50 \% / 50 \%$ & 14 & Fast & - \\
Exp7 & Snacks, fruits and vegetables & $50 \% / 50 \%$ & 10 & &
\end{tabular}

477

478 Note: Go/No-Go Proportion: the proportion of Go trials and the proportion of No-Go trials in the training. Training Length: the 479 number of repetitions for stimuli in training. Decision Speed: Fast: 1.5-second time limit; Slow: no time limit. 
481

482

483

\begin{tabular}{lccccc}
\hline \multirow{2}{*}{ Exp } & \multicolumn{2}{c}{ High Value } & \multicolumn{2}{c}{ Low Value } & \multirow{2}{*}{ Go vs. No-Go } \\
\cline { 2 - 5 } & Go & No-Go & Go & No-Go & \\
\hline Exp1 & $1.11(0.30)$ & $1.11(0.29)$ & $0.29(0.29)$ & $0.29(0.29)$ & $F(1,23)=1.38, p=0.252$ \\
Exp2 & $1.16(0.30)$ & $1.16(0.31)$ & $0.43(0.31)$ & $0.43(0.30)$ & $F(1,28)=0.01, p=0.931$ \\
Exp3 & $1.58(0.32)$ & $1.58(0.32)$ & $0.35(0.37)$ & $0.36(0.37)$ & $F(1,59)=1.87, p=0.176$ \\
Exp4 & $1.58(0.30)$ & $1.58(0.29)$ & $0.27(0.29)$ & $0.28(0.29)$ & $F(1,62)=0.09, p=0.764$ \\
Exp5 & $1.52(0.24)$ & $1.52(0.23)$ & $0.40(0.31)$ & $0.41(0.30)$ & $F(1,56)=1.58, p=0.214$ \\
Exp6 & $1.53(0.29)$ & $1.53(0.28)$ & $0.37(0.32)$ & $0.38(0.32)$ & $F(1,58)=2.46, p=0.123$ \\
Exp7 & $0.92(0.41)$ & $0.91(0.41)$ & $0.78(0.45)$ & $0.78(0.44)$ & $F(1,69)=0.15, p=0.696$ \\
\hline
\end{tabular}

484

485 Note: In Experiment 7, the high value level refers to unhealthy foods, and the low value level refers to healthy foods. Standard

486 deviations are reported in parentheses.

Table 2. Value of Go and No-Go Items Before Training

487

488 
490 Table 3. Percentages of Choosing Go Items on Experimental Trials and of Choosing High-Value Items on Filler Trials

491

\begin{tabular}{|c|c|c|c|c|c|c|}
\hline \multirow{2}{*}{$\begin{array}{c}\text { Experiment } \\
\text { (Decision Speed) }\end{array}$} & \multicolumn{3}{|c|}{ Experimental Trials } & \multicolumn{3}{|c|}{ Filler Trials } \\
\hline & HV Pairs & LV Pairs & Median Choice RT & Go Pairs & No-Go Pairs & $\begin{array}{c}\text { Median Choice } \\
\text { RT }\end{array}$ \\
\hline Exp1 (slow) & $55.1 \%(19.4 \%)$ & $50.9 \%(27.5 \%)$ & $1142.6(409.8)$ & $83.6 \%(20.8 \%)$ & $83.7 \%(20.7 \%)$ & $908.8(290.2)$ \\
\hline Exp2 (fast) & $54.2 \%(19.0 \%)$ & $58.9 \%(22.5 \%)$ & $744.9(107.9)$ & $80.1 \%(14.3 \%)$ & $78.6 \%(18.7 \%)$ & $715.7(95.9)$ \\
\hline Exp3 (slow) & $50.1 \%(19.9 \%)$ & $47.8 \%(24.0 \%)$ & $1460.0(703.4)$ & $85.9 \%(19.8 \%)$ & $90.5 \%(12.0 \%)$ & $1119.7(484.6)$ \\
\hline Exp4 (fast) & $52.0 \%(21.0 \%)$ & $59.4 \%(23.1 \%)$ & $749.0(106.2)$ & $89.8 \%(13.9 \%)$ & $89.4 \%(12.6 \%)$ & $681.6(89.8)$ \\
\hline Exp4 - Retest (fast) & $48.4 \%(21.4 \%)$ & $52.0 \%(23.0 \%)$ & $700.5(104.6)$ & $85.9 \%(15.3 \%)$ & $85.6 \%(15.1 \%)$ & $647.3(93.6)$ \\
\hline Exp5 (fast) & $59.9 \%(20.4 \%)$ & $63.7 \%(23.3 \%)$ & $742.0(115.7)$ & $87.2 \%(14.0 \%)$ & $88.4 \%(13.6 \%)$ & $691.5(100.8)$ \\
\hline Exp6 (fast) & $57.2 \%(18.3 \%)$ & $56.9 \%(24.6 \%)$ & $748.6(103.2)$ & $85.3 \%(14.9 \%)$ & $88.4 \%(13.6 \%)$ & $701.8(92.7)$ \\
\hline Exp5\&6 - Retest (fast) & $53.5 \%(21.2 \%)$ & $54.2 \%(21.3 \%)$ & $697.0(98.1)$ & $84.2 \%(16.3 \%)$ & $85.2 \%(15.4 \%)$ & $659.3(90.4)$ \\
\hline Exp7 (fast) & $62.1 \%(20.9 \%)$ & $60.8 \%(21.7 \%)$ & $704.0(142.9)$ & - & - & - \\
\hline
\end{tabular}

492

493 Note: HV Pairs: High-value pairs; LV Pairs: Low-value pairs. Choice RTs are reported in milliseconds. Standard deviations are 494 reported in parentheses. 


\section{Results}

Food Value. To investigate the effect of GNG on food reward value, we carried out a repeated-measures ANOVA on WTP before and after training, with measurement time (before

499 vs. after training), value level (high vs. low) and training condition of items (go vs. no-go) as

500 independent variables. The interaction effect between measurement time and training condition

501 of items was statistically significant, $F(1,28)=4.52, p=.043, \eta^{2}=.001$. While before the

502 training, there was no difference in WTP between go and no-go items (see Table 2), after the

503 training, the main effect of training condition was statistically significant, $F(1,28)=4.68, p=$

$504.039, \eta^{2}=.004$. Participants were willing to pay more for go items $\left(M_{\text {high }}=1.094, S D=0.40\right.$;

$\left.505 M_{\text {low }}=0.579, S D=0.42\right)$ than for no-go items $\left(M_{\text {high }}=1.080, S D=0.38 ; M_{\text {low }}=0.512, S D=\right.$

506 0.35). However, the training was not more effective for high-value items, as the interaction effect

507 between training condition and value level on post-training WTP was not statistically significant,

$508 F(1,28)=0.66, p=.424, \eta^{2}=.002$. If anything, the value difference between go and no-go

509 items was numerically larger for low-value pairs $\left(M_{\text {diff }}=0.067\right)$ than for high-value pairs $\left(M_{\text {diff }}=\right.$

510 0.014) after the training, opposite to the initial prediction.

511 Food Choices. When choosing with time limit, participants overall chose go items

512 significantly more often, mean proportion $=56.6 \%, \mathrm{OR}=1.32,95 \% \mathrm{CI}=[1.06,1.64], p=.013$.

513 Furthermore, participants chose go items more often for low-value pairs than for high-value pairs

514 (Figure 2, lower panel), but the difference was not statistically significant, $\mathrm{OR}=1.24,95 \% \mathrm{CI}=$

$515[0.73,2.11], p=.430$. On the filler trials, participants chose high-value items more often, mean

516 proportion $=79.3 \%, \mathrm{OR}=4.78,95 \% \mathrm{CI}=[3.34,6.83], p<.001$ (see Figure 3$)$. This shows that

517 even under such strict time limit (see Table 3 for median choice reaction times), participants still

518 could assess the value of the snacks and make value-based decisions. 


\section{Discussion}

520 GNG increased people's preferences for go items when they chose quickly. The initial

521 value of snacks did not seem to moderate the effect, as participants chose go items similarly

522 often for high-value pairs and for low-value pairs (if anything, they seemed to choose go items

523 more often for low-value pairs). These fast choices were meaningful choices, as participants

524 were well informed that they were choosing snacks for real consumption. In addition, on the

525 filler trials participants overall preferred high-value over low-value items, suggesting that they

526 could make value-based decisions within the strict time limit of 1.5 seconds.

527 GNG also influenced the value of go and no-go items as assessed by the auction task.

528 Participants were willing to pay more for the go snacks than for the no-go snacks after training,

529 while before the training the two were matched in WTP. Similar to the findings on choices, this

530 effect was not moderated by the reward value of items. If anything, value change induced by

531 training was larger for low-value items than for high-value items, which is not in line with the

532 BSI theory. However, since the second auction task was conducted after the choice task, this

533 change in value could either be caused by GNG, or by the choice task preceding the second

534 auction task. The current design does not allow us to disentangle these two possibilities, and we

535 will focus on the effect of GNG on choice rather than value in the remainder of this paper.

537 In Experiment 3, we aimed to repeat and extend Experiment 1, by recruiting more

538 participants, and using different stimuli and a different task for measuring value. Since the results

539 of Experiment 1 and 2 were not in line with our initial predictions, for this experiment a priori

540 we did not have directional hypotheses.

\section{$541 \quad$ Method}


Sample Size. In Experiment 3, we planned to recruit between 60 and 65 participants.

543 This increase in sample size was motivated by the observation that due to the exclusion of

544 participants, the final sample size was smaller than the planned sample size in Experiments 1 and

545 2. Doubling the sample size left more room for potential exclusion. In Experiments 1 and 2,

546 paired-samples $t$ test was used as the planned test in power analysis, whereas when analyzing the

547 choice data, repeated-measures logistic regression was used. To resolve this inconsistency, we

548 simulated data to evaluate the power of repeated-measures logistic regression with 60

549 participants. Power simulation suggested that with 60 participants, we have around 90\% power at

550 an alpha level of .05, with repeated-measures logistic regression as the planned test and Cohen's

$551 d$ of 0.5 as the expected effect size. Details on the power simulation can be found in footnote 4 .

552 Participants. Sixty participants took part in the experiment, and no participant met the

553 preregistered exclusion criterion (accuracy on go or no-go trials in the training $3 S D$ below

554 sample mean and below 90\%). For why we only used GNG performance as the exclusion

555 criterion in the current experiment, see footnote 5.

556 Materials and Procedure. Experiment 3 was similar to Experiment 1, except a few

557 changes. First, instead of using snacks, we used images of 60 different candies (e.g., gummies,

558 hard candies, nougats, chocolate etc.). All candies were purchased from local candy stores and

559 familiar to participants. Each type of candies was placed on a white plate against grey

560 background, and arranged to occupy a similar amount of area on the plate.

561 Second, instead of using an auction task, in Experiment 3 we used a rating task in which

562 participants reported how much they wanted to eat each of the candies at that moment by using a

563 200-point scale $(0=$ Not at all; 2 =Very much; see Krajbich et al., 2010, where a similar

564 question was used to probe the value of food items). Because of the small amount of candies on 
565 each image (e.g., two nougats, six gummy bears), monetary bids may not be sensitive enough to

566 the variation in reward value as participants might bid relatively low for all items. We therefore

567 used the rating task instead when candies were used as stimuli (Experiments 3-6). The auction

568 task was used again in Experiment 7 where snacks, fruits and vegetables were used as stimuli.

569 Third, in Experiments 1 and 2, all 60 images were used in the training. The GNG

570 contained more go trials (around 75\%) than no-go trials (around 25\%). In Experiment 3, only the

57132 selected images were used in the training: of which 16 were paired with no-go responses and

57216 with go responses. The GNG in Experiment 3 and all following experiments thus contained

573 an equal number of go and no-go trials (see Table 1 for an overview). This change enabled us to

574 better isolate the effect of mere action versus inaction on preferences. The selection procedure in

575 Experiment 3 was the same with the one used in Experiment 1 and 2, except that all unselected

576 items were not used. See Figures S2 in the Supplemental Materials for the selection procedure.

577 Lastly, GNG included 6 blocks (instead of 8) in Experiment 3, resulting in 192 trials in

578 total. In Experiment 3 and all the following experiments, participants did not receive a rating or

579 auction task after training. In short, in Experiment 3 participants received the following tasks in

580 order: rating task, GNG, food choice task without time limit, memory recognition task and

581 demographic questions.

582 Results

583 In line with Experiment 1, on the experimental trials, participants did not choose go items

584 more often, mean proportion $=48.9 \%, \mathrm{OR}=0.95,95 \% \mathrm{CI}=[0.79,1.13], p=.546$. The

585 difference between high-value and low-value pairs was not statistically significant, $\mathrm{OR}=0.90$,

$58695 \% \mathrm{CI}=[0.62,1.30], p=.571$. Although we used different stimuli and a different task to assess

587 food value, on the filler trials participants still preferred high-value items, mean proportion $=$ 
$88.2 \%, \mathrm{OR}=19.06,95 \% \mathrm{CI}=[11.46,31.71], p<.001$, suggesting that they still made value-

589 based choices and that self-reported wanting is a valid measure of food reward value.

590 Discussion

591 Using a larger sample size, we did not find an effect of GNG on slow choices. The

592 absence of effect in Experiment 1 was thus replicated. This again could not be explained by

593 participants' indifference or lack of motivation, as the choices on the filler trials were clearly

594 guided by the value of candies. Again, there was no significant difference between choices with

595 high-value pairs and with low-value pairs. However, to show that this absence of effect was not

596 due to any procedural changes from Experiments 1 and 2 to Experiment 3 (i.e., difference in

597 stimuli used, tasks for assessing value, length of training), but a genuine moderation effect by

598 decision time, it is important to show the effect of GNG on preferences with time limit again,

599 with the same setup. In Experiment 4, we used the setup from Experiment 3 but added the 1.5-

600 second time limit on choices, to see whether the results from Experiment 2 could be replicated.

\section{Experiment 4 - Fast Choice}

602 Experiment 4 was a replication and extension of Experiment 2. We predicted that after

603 training, participants would choose go items more often. Since no significant difference between

604 high-value and low-value pairs had been observed, we did not have a specific hypothesis

605 concerning whether the reward value of items would moderate the effect.

606 Method

607 Sample Size. As in Experiment 3, we planned to recruit between 60 and 65 participants.

608 Participants. Sixty-three participants took part in the experiment, and none of them met

609 the preregistered exclusion criterion (accuracy on go or no-go trials in the training $3 S D$ below

610 sample mean and below 90\%). 
6123 , except that in the choice task participants had 1.5 seconds for each choice.

\section{Results}

614 Replicating the results of Experiment 2 and in line with our prediction, participants chose 615 go items more often on experimental trials, mean proportion $=55.7 \%, \mathrm{OR}=1.28,95 \% \mathrm{CI}=$

$616[1.08,1.52], p=.005$. Descriptively, the effect was stronger for low-value pairs than for high-

617 value pairs, but the difference was not statistically significant, $\mathrm{OR}=1.40,95 \% \mathrm{CI}=[0.97,2.03]$,

$618 p=.074$. On the filler trials, participants again chose high-value items more often, mean

619 proportion $=89.6 \%, \mathrm{OR}=12.92,95 \% \mathrm{CI}=[9.55,17.47], p<.001$.

\section{Discussion}

Using a larger sample size and a slightly different procedure, results from Experiment 2

622 were replicated: participants preferred go items when choosing under time pressure. Although

623 not in line with our predictions (we predicted an effect for slow choices with high-value items in

624 Experiment 1), the results from the first four experiments were quite consistent. GNG changed

625 people's preferences when they chose quickly, but not when they had more time to decide.

626 Furthermore, item reward value did not seem to moderate the effect. Direct comparisons between

627 high-value and low-value pairs did not show significant difference in all four experiments. In the 628 remaining experiments, we therefore did not have directional hypotheses for item reward value.

629 The effect of item reward value will be explored in the final exploratory analyses with data from 630 all experiments combined.

\section{Experiment 4 Retest - Fast Choice}

632 In all four experiments presented till now, participants received the choice task

633 immediately after training. It was unclear whether the training-induced preference change would 
634 still be observed after some delay. To explore this question and estimate the effect size of

635 potential decay, participants from Experiment 4 were invited back to the lab at least one week

636 after training, and received the same choice task again (i.e., with time limit of 1.5 seconds). This

637 experiment was exploratory in nature and we did not have directional hypotheses.

638 Method

639 Sample Size. Sample size could not be determined a priori as it depended on how many

640 of the 63 participants from Experiment 4 would respond to the invitation for the retest session.

641 Participants. In total, 47 participants responded to the invitation and took part in the

642 retest session. The average delay between the training and the retest session was 12.4 days $(S D=$

6433.1 , range $=[7,19])$.

644 Materials and Procedure. Participants were required to not eat anything for at least 3

645 hours. They received a same food choice task as the one they received in the first session, with

646 1.5-second time limit. After the choice task, they received the same memory recognition task,

647 and reported their current hunger level $(-100=$ Not hungry at all; $100=$ Very hungry $)$ and hours

648 since the last time of food consumption. They then received one bag of candies based on a trial

649 selected from the choice task, and were compensated and thanked.

650 Results

651 Participants did not choose go items more often on the experimental trials, mean

652 proportion $=50.2 \%, \mathrm{OR}=1.01,95 \% \mathrm{CI}=[0.83,1.22], p=.959$. This proportion of go choices

653 was significantly smaller than that immediately following training, $\mathrm{OR}=0.86,95 \% \mathrm{CI}=[0.76$,

$6540.98], p=.024$. For an exploratory analysis on the decay of the effect as a function of the delay

655 between two sessions, see the Supplemental Materials. The difference between high-value and

656 low-value pairs was again not statistically significant, $\mathrm{OR}=1.18,95 \% \mathrm{CI}=[0.77,1.83], p=$ 
657.450 . On the filler trials, participants still preferred high-value items, mean proportion $=85.7 \%$,

$658 \mathrm{OR}=8.39,95 \% \mathrm{CI}=[6.09,11.57], p<.001$.

659 To explore the consistency of choices on experimental trials between two sessions,

660 choices in session 1 (i.e., whether the chosen item was a go or no-go item) were used to predict

661 choices in session 2 with a repeated-measures logistic regression. For the trials where

662 participants chose go items in session 1, they were more likely to choose go items again in

663 session 2 (mean proportion of go choices $=69.5 \%, S D=15.3 \%$ ), compared to the trials where

664 they chose no-go items in session 1 (mean proportion of go choices $=27.4 \%, S D=14.0 \%$ ), OR

$665=7.40,95 \% \mathrm{CI}=[5.83,9.40], p<.001$. In other words, on average participants made the same

666 choices for about $70 \%$ of the trials, suggesting that their choices were fairly consistent.

\section{Discussion}

668 By administering the same choice task to participants one week after training, we

669 explored to what extent the effect of GNG on preference was durable. Overall, the training effect

670 showed a large decrease, to the extent that participants did not choose go items more often in the

671 retest session. This seemed to suggest that GNG did not have durable influence on preference.

672 However, the consistency in choices revealed by the exploratory analysis suggested that

673 preference change after one week might be observed if we could (1) increase the effect size of

674 training-induced preference change and (2) increase sample size to have sufficient power for

675 detecting a small effect. In Experiment 4, all items were repeated 6 times in training. One way to

676 increase the effectiveness of training may be to increase the number of repetitions in training. In

677 the next two experiments we increased both the number of repetitions and the sample size to (1)

678 explore the effect of training length on the effectiveness of training and (2) see whether the

679 training could influence delayed preference with more statistical power. 


\section{Experiment 5 \& 6 - Fast Choice}

682 effect of GNG on fast choices with two more replications; (2) to explore whether the number of 683 repetitions in training would influence its effectiveness, by presenting each item 10 times in 684 GNG in Experiment 5 and 14 times in Experiment 6; (3) to examine the delayed effect with a 685 larger sample. We predicted that participants would prefer go items immediately after training, in 686 both Experiment 5 and 6. Anticipating a small delayed effect, we a priori decided to combine 687 data from both experiments when examining the delayed effect. For this combined dataset, we 688 predicted that participants would choose go items more often than no-go items.

\section{Method}

690 Sample Size. We planned to recruit between 60 to 65 participants for each experiment. 691 Participants. Sixty participants took part in Experiment 5, of which 3 were excluded 692 based on preregistered exclusion criterion (accuracy on go or no-go trials in the training $3 S D$ 693 below sample mean and below 90\%). The final sample consisted of 57 participants. Sixty 694 participants took part in Experiment 6, of which 1 was excluded (same exclusion criterion as in 695 Experiment 5). The final sample consisted of 59 participants.

696 Materials and Procedure. The procedure of Experiments 5 and 6 was the same as 697 Experiment 4, except that the GNG contained 10 blocks in Experiment 5, and 14 blocks in 698 Experiment 6.

699 Results

700 As predicted, in both Experiment 5 and 6, participants chose go items more often on 701 experimental trials, mean proportion $=61.8 \%, \mathrm{OR}=1.74,95 \% \mathrm{CI}=[1.40,2.16], p<.001$, and 702 mean proportion $=57.0 \%, \mathrm{OR}=1.37,95 \% \mathrm{CI}=[1.13,1.65], p=.001$, respectively. The 
703 differences between low-value pairs and high-value pairs were not statistically significant, $\mathrm{OR}=$

$7041.37,95 \% \mathrm{CI}=[0.92,1.83], p=.137$ in Experiment 5 and $\mathrm{OR}=1.05,95 \% \mathrm{CI}=[0.74,1.49], p=$

705.781 in Experiment 6 . They also preferred high-value items on the filler trials, mean proportion $=$

$70687.8 \%, \mathrm{OR}=10.74,95 \% \mathrm{CI}=[7.78,14.84], p<.001$ in Experiment 5, and mean proportion $=$

$70786.9 \%, \mathrm{OR}=8.79,95 \% \mathrm{CI}=[6.77,11.41], p<.001$ in Experiment 6.

708 Directly comparing Experiment 5 with Experiment 4 showed that participants in

709 Experiment 5 chose go items more often than those in Experiment 4, OR $=1.35,95 \% \mathrm{CI}=[1.03$,

$7101.77], p=.029$. However, further increasing the number of repetitions did not increase the effect

711 size further. If anything, participants chose go items a bit less often in Experiment 6 than in

712 Experiment 5, OR $=0.79,95 \% \mathrm{CI}=[0.60,1.04], p=.093$. There was also no significant

713 difference between Experiment 6 and 4, $\mathrm{OR}=1.07,95 \% \mathrm{CI}=[0.82,1.39], p=.631$.

714 Discussion

715 In line with our predictions and the results from Experiments 2 and 4, the effect of GNG

716 on fast choices immediately following training was replicated when participants received longer

717 training. Although increasing the number of stimulus repetition from 6 to 10 increased the

718 training effect size, further increasing it to 14 did not make the training more effective. Next, we

719 present results from the retest session, combining data from both Experiments 5 and 6.

720

Experiment 5 \& 6 Retest - Fast Choice

\section{Method}

722 Participants. One hundred and fifteen participants took part in the retest session.

723 Average delay between the two sessions was 8.2 days $(S D=1.8$, range $=[7,14])$.

724 Materials and Procedure. The procedure was the same with the retest session of

725 Experiment 4. 


\section{Results}

In line with our prediction, participants preferred go items on the experimental trials,

728 mean proportion $=53.8 \%, \mathrm{OR}=1.18,95 \% \mathrm{CI}=[1.04,1.34], p=.009$. For the delayed effect for

729 Experiment 5 and 6 separately, see the Supplementary Materials. This delayed effect was again

730 significantly smaller than the immediate effect, $\mathrm{OR}=0.78,95 \% \mathrm{CI}=[0.72,0.84], p<.001$.

731 Participants' choices were fairly consistent. They selected go items more often in the retest

732 session if they had chosen the go items in session 1 (mean proportion $=71.3 \%, S D=13.6 \%$ ),

733 than if they had chosen no-go items before (mean proportion $=30.4 \%, S D=17.1 \%$ ), $\mathrm{OR}=7.39$,

$73495 \% \mathrm{CI}=[6.25,8.72], p<.001$. Comparison between high-value pairs and low-value pairs

735 revealed no statistically significant difference, $\mathrm{OR}=1.04,95 \% \mathrm{CI}=[0.82,1.32], p=.757$. On

736 the filler trials, high-value items were still chosen more often, mean proportion $=84.7 \%, \mathrm{OR}=$

$737 \quad 7.57,95 \% \mathrm{CI}=[6.19,9.27], p<.001$.

738 Discussion

739 As predicted, by increasing the number of stimulus repetition in training and using a

740 larger sample, we observed an effect of training on preference one week after training. The effect

741 of GNG on preference therefore seems durable and not limited to immediately following training.

742 Admittedly, the effect size was quite small after one week, and again a significant reduction in

743 effect size was observed, showing that the effect of training has decayed with the passage of time.

744 In all experiments presented so far, participants were trained to respond to certain high-

745 calorie food items (e.g., snacks, candies) and not respond to other high-calorie food items. In the

746 choice task after training, they chose between two high-calorie food items that were matched on

747 value. This procedure enabled us to isolate the effect of GNG from some confounding factors

748 (e.g., type of food, difference in reward value), but also left an important question unanswered. 
749 That is, whether GNG could be used to promote healthy choices, when people choose between

750 healthy and unhealthy foods. Examining this question is not only interesting from an applied

751 perspective, but may also extend the effect of training to a situation where the value of food

752 items is not matched, a situation that has not been examined in the previous experiments.

\section{Experiment 7 - Promoting Healthy Choices?}

754 In Experiment 7, participants were trained to respond to certain healthy (i.e., fruits and

755 vegetables) and unhealthy food items, and to not respond to other healthy and unhealthy food

756 items in GNG. After training, they chose between healthy and unhealthy items (among other

757 choices) for consumption, again within 1.5 seconds. We predicted that participants would choose

758 healthy items more often, when the healthy items were associated with go responses and

759 unhealthy items with no-go responses (i.e., healthy-trained trials), in comparison to when both

760 items were not included in the training (i.e., untrained trials). Note that the untrained choices

761 were included as a baseline to which the healthy-trained choices will be compared. This

762 modification was introduced because a priori the average value of healthy and unhealthy items

763 cannot be matched, hence the choices cannot be compared to the $50 \%$ chance level as in previous

764 experiments. We also included choices in which the unhealthy items were paired with go

765 responses and the healthy items with no-go responses (i.e., unhealthy-trained trials). These trials

766 were included to make go and no-go responses toward healthy and unhealthy items equally likely.

767 For the comparison between unhealthy-trained and untrained choices, we did not have

768 directional hypothesis, as the preexisting preference for unhealthy items might make any further

769 increase in unhealthy choices difficult to observe (i.e., ceiling effect). Lastly, participants also

770 made choices between two healthy items and between two unhealthy items, with one paired with

771 go responses and the other with no-go responses. As in previous experiments, the average value 
772 of these go and no-go items were matched. For these choices, we expected to replicate previous

773 results, such that participants would prefer go items for these choices.

\section{Method}

775 Participants. For Experiment 7, we planned to recruit 72 participants, which exceeded

776 the sample sizes used in previous experiments and left room for potential exclusion. Seventy-

777 three participants participated. Three participants were excluded based on predetermined

778 exclusion criteria (1. participants who bid less than 25 cents on more than 40 food items in the

779 first auction task; 2. participants whose accuracy on go or no-go trials was $3 S D$ below sample

780 mean and below 90\%), leaving 70 participants in the final sample.

781 Materials. Thirty unhealthy high-calorie snacks were selected from Experiments 1 and 2.

782 Thirty low-calorie food items that were generally considered healthy (i.e., fruits and vegetables;

783 Tilman \& Clark, 2014) were selected from previous work (Veling, Chen, et al., 2017).

784 Procedure. Participants were asked to fast for 3 hours before the experiment started.

785 Upon arrival, they received two euros to bid on each of the 60 food items (30 healthy and 30

786 unhealthy, mixed and randomized). After the auction, the program rank ordered all items from

787 the highest WTP to the lowest, for healthy and unhealthy foods separately. Food items were then

788 selected into different training conditions based on rankings. Eight healthy and eight unhealthy

789 items (ranked from 12 to 19 within the healthy and unhealthy category) were selected into the

790 within-category choice trials. Half of the eight selected items were assigned into the go condition,

791 whereas the remaining half was assigned into the no-go condition. These within-category choices

792 were the same with the experimental choices participants in previous experiments received. On

793 the within-category choice trials, participants chose between two healthy or two unhealthy items

794 with matched WTP, one paired with go responses and the other with no-go responses. 
Eighteen healthy and eighteen unhealthy items (ranked from 3 to 11 and from 20 to 28 within the healthy and unhealthy category) were selected for the between-category choices (i.e.,

797 choices between healthy and unhealthy items). Crucially, the between-category choices were

798 further divided into three conditions: healthy-trained condition, in which healthy go item was

799 paired with unhealthy no-go item; unhealthy-trained condition, in which healthy no-go item was

800 paired with unhealthy go item; and untrained condition, in which both the healthy and unhealthy

801 item were not used in the training but included in the choice task. Note that the unhealthy-trained

802 condition was included to ensure an equal number of healthy and unhealthy items in both go and

803 no-go trials. This made it difficult to infer the structure of GNG, and ensured that healthy choices

804 could not simply be explained by demand characteristics. When used in applied settings, these

805 unhealthy-trained trials should be omitted to avoid the promotion of unhealthy food choices.

806 After the selection procedure (for the exact selection procedure see Figures S3 and S4 in

807 the Supplemental Materials), the 40 selected items were used in GNG and repeated 10 times.

808 After the training, participants received a choice task with 1.5-second time limit. The choice task

809 consisted of 32 choices between two healthy items, 32 choices between two unhealthy items, 36

810 unhealthy-trained choices, 36 healthy-trained choices, and 36 untrained choices. Different types

811 of choices were mixed and presented in a random order. After the choice task, participants

812 received a memory recognition task and filled out some demographic questions. Results from the

813 choice task and the auction task were revealed, and participants received one or two food items.

814 They were debriefed, compensated and thanked.

\section{$815 \quad$ Results}

816 Food Value. For the result of the selection procedure for items used in within-category

817 choices, see Table 2 . To check the selection procedure for items used in between-category 
818 choices, the average WTP of the items was calculated, for healthy and unhealthy items separately.

819 The WTP was submitted to repeated-measures ANOVA, with item category (healthy vs.

820 unhealthy) and choice trial type (healthy-trained, unhealthy-trained vs. untrained) as independent

821 variables. There was a small non-significant difference between healthy and unhealthy items,

822 showing that participants were willing to pay more for unhealthy items, $F(1,69)=2.93, p=$

$823.091, \eta^{2}=.016$. Crucially, there was no significant interaction with choice trial type, $F(2,138)=$

$8240.33, p=.721, \eta^{2}<.001$, suggesting that the value difference between healthy and unhealthy

825 items was matched across different types of choices. In other words, any change in preference

826 could not be explained by preexisting value difference between healthy and unhealthy items.

827 Within-Category Choices. As predicted, and replicating previous findings, for within-

828 category choices in which participants chose between go and no-go items (both healthy or both

829 unhealthy, with matched WTP), overall they preferred go items, mean proportion $=61.5 \%, \mathrm{OR}=$

$8301.69,95 \% \mathrm{CI}=[1.41,2.03], p<.001$. This effect was found for both unhealthy items, mean

831 proportion $=62.1 \%, \mathrm{OR}=1.85,95 \% \mathrm{CI}=[1.43,2.39], p<.001$, and healthy items, mean

832 proportion $=60.8 \%, \mathrm{OR}=1.74,95 \% \mathrm{CI}=[1.33,2.29], p<.001$ (Figure 4, left panel).

833 Between-Category Choices. To compare healthy choices between healthy-trained and

834 untrained choice trials, a repeated-measures logistic regression was conducted, with choice trial

835 type as the predictor and whether the chosen item was a healthy item or not as the outcome. As

836 predicted, the main effect of choice trial type was significant, $\mathrm{OR}=1.63,95 \% \mathrm{CI}=[1.25,2.12]$,

$837 p<.001$, indicating that participants chose healthy items more often on healthy-trained trials

838 (mean proportion of healthy choices $=46.2 \%$ ) than on untrained trials (mean proportion of

839 healthy choices $=39.4 \%$; see Figure 4, right panel). Similarly, although we did not predict the 
840 effect, people chose healthy items less often in unhealthy-trained trials (mean proportion of

841 healthy choices $=32.6 \%$ ) than on untrained trials, $\mathrm{OR}=0.66,95 \% \mathrm{CI}=[0.48,0.91], p=.010$.
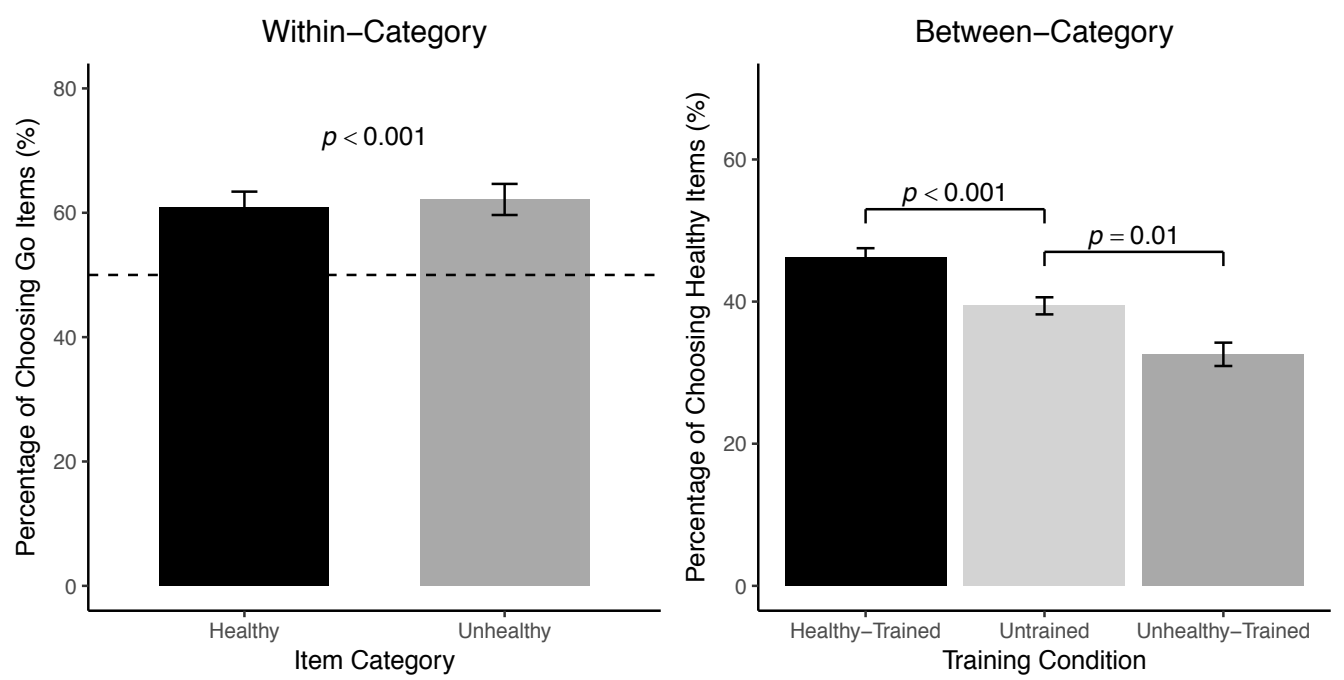

843 Figure 4. Probability of choosing go items on within-category choices (left panel) and

844 probability of choosing healthy items on between-category choices (right panel) in Experiment 7.

$845 \mathrm{P}$ values are calculated from repeated-measures logistic regression. Error bars in the left panel

846 stand for standard errors of mean proportions; error bars in the right panel stand for within-

847 subject standard errors of mean.

849 Discussion

850 Experiment 7 replicated and extended the previous findings, by showing that GNG

851 modified not only choices between unhealthy items, but also choices between healthy items.

852 Moreover, the training also promoted healthy food choices, when people chose between healthy

853 and unhealthy items. Note that the promotion of healthy food choices by GNG is in a relative

854 sense, but not in an absolute sense. That is, although in all three between-category choice

855 conditions, participants chose healthy items less than $50 \%$ of the time (see Figure 4, right panel),

856 their preference for healthy items increased when they chose between a healthy go item and an 
857 unhealthy no-go item, in comparison to when both items were untrained (i.e., the increase of

858 healthy choices from untrained condition to healthy-trained condition; see Figure 4, right panel).

859 Although the effect of GNG on choices between healthy and unhealthy items has been

860 shown before (Porter et al., 2018; Veling et al., 2013b), the current finding is still noteworthy

861 because of the methodological improvement (i.e., repeated choices for real consumption rather

862 than one-shot hypothetical choice). Furthermore, the inclusion of different types of choices and

863 the 1.5-second time limit (which were not used in previous work) makes it very unlikely that the

864 observed results were due to demand characteristics. The current finding therefore serves as a

865 strong demonstration that GNG can promote healthy food choices.

866 Furthermore, this finding also suggests that the effect of GNG on preference is not

867 limited to situations where the value of two choice options is closely matched. Rather, the

868 training seems able to overcome value difference (at least to some extent) and increase choices

869 for relatively low-value items. Although no filler choice trials were included, the choices

870 participants made were arguably still value-based, as the overall proportion of healthy choices

871 was below 50\%, which mirrors the finding that the average WTP was lower for healthy items

872 than for unhealthy items. The value difference between healthy and unhealthy items was

873 admittedly small and statistically not significant. Future research may look into whether the

874 training changes preference when the value difference is more substantial.

875 In all experiments reported so far, we consistently found that GNG changed preference,

876 but only when participants chose under time limit. Furthermore, item reward value did not seem

877 to moderate the effect. Next, we conducted exploratory analyses on combined datasets to further

878 explore whether and how decision time and item reward value may moderate the effect. 
To directly compare fast choices with slow choices, data from all choice tasks

881 immediately after training were combined (i.e., Experiments 1-3, 7 and the first session of

882 Experiments 4-6). Only the trials on which people chose between two similarly valued go and

883 no-go items were included in this analysis. Multilevel logistic regression was used, with trials

884 nested within participants, and participants nested within experiments. Overall, when participants

885 had unlimited time to make choices (Experiments 1, 3; 84 participants, 5376 trials), they did not

886 choose go items significantly more often than no-go items, mean proportion $=50.0 \%, \mathrm{OR}=1.00$,

$88795 \% \mathrm{CI}=[0.86,1.16], p=.997$. However, when participants chose within 1.5 seconds

888 (Experiments 2, 4-7; 278 participants, 17792 trials), they chose go items more often for

889 consumption, mean proportion $=59.6 \%, \mathrm{OR}=1.48,95 \% \mathrm{CI}=[1.32,1.66], p<.001$. The

890 difference between fast and slow choices was statistically significant, $\mathrm{OR}=1.47,95 \% \mathrm{CI}=[1.18$,

$8911.82], p=.001$. These results thus suggest that GNG more effectively influenced fast choices

892 than slow choices. Note that based on these results, it is premature to conclude that slow choices

893 cannot be changed by GNG. In the two experiments on slow choices reported here (Experiment 1

894 and 3), a relatively small number of stimulus repetitions were used. The possibility remains that

895 with a more extensive training by repeating the stimuli more times, choices made without time

896 limit may eventually also be changed. We are currently conducting another line of research that

897 may provide more insights into this question.

898 One and a half seconds was used as an arbitrary cut-off value to distinguish between fast

899 and slow choices. However, decision time varies on a continuum. To gain a more nuanced

900 understanding of how decision time influences the effect, we carried out an exploratory analysis

901 by using decision time as a continuous predictor. To reduce the influence of extreme values, for

902 each participant choice trials with extreme reaction times were first removed (more than 2.5 
903 absolute deviation from the median; Leys, Ley, Klein, Bernard, \& Licata, 2013). For the

904 remaining trials, decision time was standardized for each participant and used as predictor for

905 choices. All random intercepts and random slopes were included. For participants who received

906 the choice task with time limit, longer decision time was associated with fewer choices for go

907 items, $\mathrm{OR}=0.88,95 \% \mathrm{CI}=[0.84,0.92], p<.001$. For those who received the choice task

908 without time limit, the effect of decision time was not statistically significant, OR $=0.96,95 \%$

$909 \mathrm{CI}=[0.87,1.06], p=.387$. Interestingly, for the retest session in which participants chose with

910 time limit, longer decision time was again related to lower likelihood of choosing go items, OR =

$9110.91,95 \% \mathrm{CI}=[0.85,0.96], p=.002($ see Figure 5$)$. The pattern of the results remained the same

912 when choice trials with extreme decision times were included.
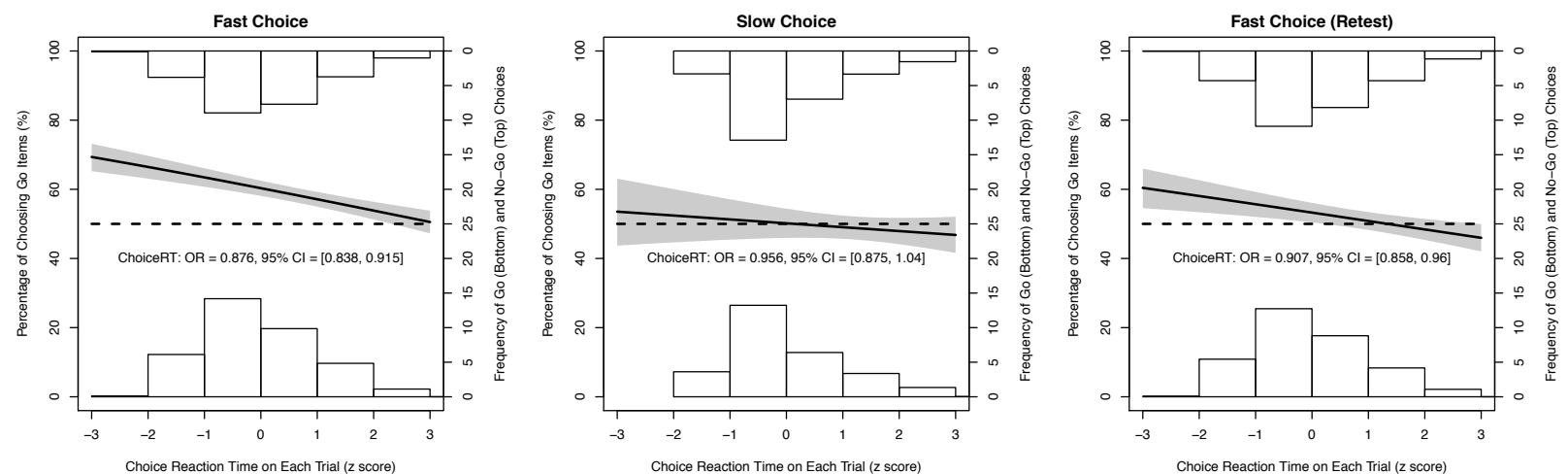

913

914 Figure 5. Probability of choosing Go items predicted by decision time (standardized). Trials with

915 extreme reaction times were removed (more than 2.5 absolute deviation from the median).

916 Shaded region stands for $95 \%$ confidence interval. Heights of bars stand for the average

917 frequencies of choosing Go items (bottom) and choosing No-Go items (top) within each reaction

918 time bin.

919

\section{Exploratory Analyses on Reward Value}

920

In the first six experiments, the effect of GNG on preference was not significantly

921 moderated by item reward value. To more reliably assess whether GNG more effectively 
922 changed preferences for high-value items, we combined the choice data from Experiments 1 to 6.

923 Data from Experiment 7 were not included, as item reward value was not manipulated in

924 Experiment 7. Multilevel analysis was used, with trials nested within participants, and

925 participants nested within experiments. Whether the two items presented were of low or high

926 value was used as the predictor, and whether the chosen item was a go or no-go item was used as

927 the outcome. All random intercepts and random slopes were included. For experiments in which

928 participants made choices with time limit (i.e., Experiments 2, 4-6; 208 participants), they chose

929 go items less often when both items were of high value compared to when both items were of

930 low value, $\mathrm{OR}=0.81,95 \% \mathrm{CI}=[0.66,0.98], p=.029$. This effect, however, was not found for

931 delayed choices (i.e., retest session of Experiments 4-6; 162 participants), $\mathrm{OR}=0.93,95 \% \mathrm{CI}=$

$932[0.74,1.15], p=.491$, and for choices without time limit (i.e., Experiments 1 and 3; 84

933 participants), $\mathrm{OR}=1.14,95 \% \mathrm{CI}=[0.81,1.60], p=.443$.

934 A key assumption of the BSI theory is that the strength of response conflict is jointly

935 determined by the initial approach tendency and ensuing response inhibition process. In the

936 current research, we selected items based on participants' monetary bids or self-reported wanting,

937 and assumed high-value items evoked stronger impulses than low-value items. To directly test

938 this assumption, items that were used in the experimental choice trials were selected for each

939 participant, and go and no-go trials involving these items were selected from GNG. The results

940 showed that compared to low-value items, participants responded to high-value items more

941 accurately on go trials, $t(291)=2.66, p=.008$, Hedges's $g=0.155,95 \% \mathrm{CI}=[-0.008,0.318]$,

942 and less accurately on no-go trials, $t(291)=-4.20, p<.001$, Hedges's $g=-0.245,95 \% \mathrm{CI}=[-$

$9430.409,-0.082]$. Furthermore, on go trials they responded to high-value items more quickly

944 compared to low-value items, $t(291)=-7.45, p<.001$, Hedges's $g=-0.435,95 \% \mathrm{CI}=[-0.600,-$ 
9450.271 ] when using median reaction time, and $t(291)=-8.57, p<.001$, Hedges's $g=-0.501,95 \%$

$946 \mathrm{CI}=[-0.666,-0.336]$ when using mean reaction time. These results are in line with previous

947 findings (Chen, Veling, Dijksterhuis, et al., 2018a), and suggest that the high-value items indeed

948 triggered stronger impulses to respond than low-value items. However, the impulse-invoking

949 quality of high-value items does not seem to lead to stronger preference change for these items.

\section{General Discussion}

951 In the current research, we conducted 7 preregistered experiments to investigate when

952 linking mere action versus inaction to objects would lead to preference change. Three questions

953 concerning training-induced preference change were examined, namely the influence of decision

954 speed, the durability of the effect and the role of item reward value. GNG reliably led to

955 preference change when participants made choices within 1.5 seconds (Experiments 2, 4-7), but

956 not when they chose without time limit (Experiments 1 and 3). Within fast choices, they were

957 more likely to choose go items on trials where they chose quickly. Preference change induced by

958 GNG was still observed one week later, although the effect size largely decreased compared to

959 immediately after training. High-value items seemed to invoke stronger go responses than low-

960 value items, but this did not translate into larger preference change for high-value items. Next we

961 discuss the implications of these findings in turn.

\section{Decision Speed}

963 Contrary to our initial prediction, preference change induced by GNG seems to be

964 constrained to fast choices. By repeatedly responding to certain food items and withholding

965 responses toward other items, participants may form associations between food items and simple

966 motor responses (Logan, 1988; Verbruggen et al., 2014) and/or associations between foods items

967 and affective reactions that accompany the responses. For instance, withholding responses may 
968 trigger negative affect (Chen et al., 2016; Veling et al., 2008), while responding to food items

969 may elicit positive affect (Guitart-Masip et al., 2014), and the affective reactions may become

970 attached to food items. Although the exact content of the acquired associations is not clear (i.e.,

971 whether the food items are associated with simple motor responses or with affective reactions), it

972 seems that the acquired associations can be rapidly retrieved and influence people's choices

973 when they choose quickly under time pressure (Berkman et al., 2017; Forstmann et al., 2016;

974 Strack \& Deutsch, 2004). When more decision time is allowed, the transient activation of the

975 learned associations may quickly decay. More decision time may allow for the incorporation of

976 more information into the construction of preferences (Sullivan et al., 2015). As more

977 information is incorporated into a decision process, the influence of the initially retrieved

978 associations may weaken.

979 Interestingly, reliable effects of GNG on evaluation have been found when evaluation

980 was assessed with self-paced explicit rating (Chen et al., 2016; Chen, Veling, Dijksterhuis, et al.,

981 2018a; Lawrence, Sullivan, et al., 2015; Serfas, Florack, Büttner, \& Voegeding, 2017a; Veling et

982 al., 2008). Evaluation is thus susceptible to the influence of GNG when people rate objects

983 without time pressure, whereas preferences are influenced by GNG when people choose quickly

984 under time pressure. One explanation for this inconsistency can be that the thoroughness of

985 information processing may differ between evaluating and choosing food items. When

986 evaluating a food item, people may sample as minimal information as possible, as the accuracy

987 of the evaluation does not have any immediate behavioral consequences. Thus, the affective

988 reactions or the response tendencies triggered by a food item may be sufficient input for one to

989 arrive at an evaluation. More decision time does not lead to the revision of this initial immediate

990 evaluation, as people may not be motivated to take more information into account (e.g., the 
991 iterative reprocessing model of evaluation; Cunningham \& Zelazo, 2007; Cunningham, Zelazo,

992 Packer, \& Van Bavel, 2007). In contrast, when choosing for real consumption, people are more

993 likely to consider all relevant information thoroughly when there is sufficient time. The initial

994 preference for go items induced by GNG may thus decrease when other information (e.g., the

995 caloric content of each food, what one ate before etc.) enter the decision process.

996 Essentially, this explanation suggests that decision time itself is not crucial; rather, the

997 effect of GNG on preference depends on whether more information is integrated into a decision

998 process to reduce the influence of GNG. In that case, factors other than decision time that also

999 influence the incorporation of information can also moderate the effect of GNG, such as people's

1000 motivation to consider all information (as discussed above) and the opportunity afforded by the

1001 situation, such as whether they are under cognitive load or not (Fazio, 1990). These questions

1002 need to be explored to further understand how different factors, including decision time,

1003 influence the training effects.

1004 The current finding also raises new questions on how to interpret previous findings

1005 showing effects of GNG on more distal outcomes such as eating behavior or weight loss that did

1006 not manipulate time pressure. For instance, previous work has found that GNG can facilitate

1007 weight loss attempts (Lawrence, Sullivan, et al., 2015; Veling et al., 2014). Based on the present

1008 finding, it seems possible that the training may have contributed to weight loss by changing

1009 people's fast food decisions, but not or less so when people spend more time to carefully think

1010 about what food items to choose. This hypothesis is difficult to test, but has important

1011 implications for the application of GNG. For instance, GNG may not work well in tandem with

1012 interventions that aim to facilitate weight loss by promoting deliberative decision-making. This

1013 may explain why combining GNG with an implementation intention intervention (Gollwitzer, 
1014 1999) that reminds people about their weight loss goals so that they are more likely to carefully

1015 think about their food choices did not lead to an additive effect on weight loss compared to using

1016 one of the two interventions alone (Veling et al., 2014).

1017 Lastly, the finding that effects on preferences are only observed under time pressure

1018 argues against the possibility that participants merely apply a simple rule to choose go items

1019 during the choice task. The accurate application of a rule would likely benefit from having more

1020 time, which would lead to larger preference change with longer decision time. This reasoning

1021 suggests preference changes induced by GNG are unlikely due to demand characteristics to

1022 choose go items over no-go items.

1023 The Durability of Preference Change

1024 In line with a previous meta-analysis that estimated the effects of GNG across time across

1025 different studies (Allom et al., 2016), the effect of GNG on preferences shows a large decrease in

1026 effect size from immediately after training to one to two weeks later. Importantly, by using the

1027 same behavioral measure to assess effects over time, the present finding shows that the lack of

1028 longevity of GNG-induced effects observed in previous work (Allom et al., 2016) is not simply

1029 an artifact of the different behavioral measures used. Rather, behavior change induced by GNG

1030 may indeed by relatively short-lived.

1031 In contrast to the relatively short-term effect of GNG, CAT has been shown to induce

1032 highly durable preference changes, up to six months after training (Salomon et al., 2018). Some

1033 procedural features of CAT distinguish it from GNG, such as the inclusion of more no-go trials

1034 than go trials, the absence of no-go cues (participants are asked to respond to go cues and not

1035 respond when no cue is presented), and the dynamic adjustment of the delay between stimulus

1036 onset and the go cues (go cues are presented such that participants manage to respond in time on 
1037 approximately $75 \%$ of the trials). Because of these procedural features, preference change

1038 induced by CAT is often not explained by simple motor responses, but by heightened attention

1039 that accompanies the execution of go responses (Bakkour et al., 2016; Schonberg et al., 2014). In

1040 GNG, go trials and no-go trials are equally frequent and both go and no-go cues are presented,

1041 which allows us to examine the effect of mere action versus inaction without other potentially

1042 confounding factors. The current findings therefore serve as a strong demonstration that merely

1043 responding or not responding to objects can change people's preferences.

1044 In terms of the longevity of the training-induced preference change, GNG and CAT show

1045 quite different patterns. Here we explored the effect of GNG one to two weeks after training, a

1046 time frame much shorter than the ones explored in previous work on CAT (from one to six

1047 months; Salomon et al., 2018; Schonberg et al., 2014). However, after this relatively short delay,

1048 preference change by GNG already shows a large decrease. For comparison, the effect of GNG

1049 one to two weeks post-training is numerically smaller than that obtained with CAT six months

1050 post-training (Salomon et al., 2018). This large difference in the durability of the effects is

1051 consistent with the argument that CAT and GNG lead to preference change via different

1052 underlying mechanisms. CAT therefore seems more suitable for long-term preference change.

1053 Future work is needed to better understand the different natures of the associations learned

1054 during CAT and GNG, and how these associations lead to sustainable changes in preferences.

\section{The Role of Reward Value}

1056 We originally predicted that GNG would lead to preference change only for high-value

1057 items when participants made choices without time limit, as only high value items require strong

1058 inhibition of impulsive responses once a no-go cue is presented (Veling et al., 2008). Contrary to

1059 this expectation, we find that GNG more strongly changed preference when participants made 
1060 choices with time limit, and the effect was not stronger for high-value items. This unexpected

1061 result leads to interpretational difficulties with explaining how preferences were changed. For

1062 instance, it could be that stimulus-stop associations have been acquired for both high- and low-

1063 value items, and that these associations influence preferences for high- and low-value food items

1064 alike. Alternatively, it could be that high-value no-go items were devalued (Chen et al., 2016;

1065 Serfas, Florack, Büttner, \& Voegeding, 2017b; Veling et al., 2008), while low-value go items

1066 increased in value (Chen et al., 2016; Chen, Veling, Dijksterhuis, et al., 2018a). The underlying

1067 mechanisms for preference change may differ between high- and low-value items, but the

1068 behavioral outcome can remain the same. The current data do not allow us to disentangle these

1069 different accounts.

1070 Implications for Applied Behavioral Interventions

1071 Tasks that manipulate simple responses (including GNG) have been shown to influence a

1072 range of behavioral outcomes, such as volume of consumption (Bowley et al., 2013; Folkvord,

1073 Veling, \& Hoeken, 2016; Houben, 2011; Houben, Havermans, Nederkoorn, \& Jansen, 2012;

1074 Houben \& Jansen, 2011, 2015; Jones \& Field, 2013; Lawrence, Verbruggen, et al., 2015; Veling

1075 et al., 2011), and self-selected portion size (Van Koningsbruggen, Veling, Stroebe, \& Aarts,

1076 2014). Multiple sessions of training have even been shown to facilitate weight loss in two

1077 samples (Lawrence, Sullivan, et al., 2015; Veling et al., 2014; see also Stice, Yokum, Veling,

1078 Kemps, \& Lawrence, 2017). Manipulating simple responses toward objects therefore seems

1079 feasible as a behavior change intervention.

1080 In light of the interest of using these tasks in applied settings, we explored two questions

1081 that are important from an applied perspective. First, we varied the number of stimulus repetition

1082 in training (Experiments 4-6) to observe its influence on the effect. Although initially increasing 
1083 the number of repetition increases the effectiveness of training, further lengthening the training

1084 does not lead to more effectiveness. This may be because learning during training is a function of

1085 both the number of repetitions and the amount of attention allocated to the training. While

1086 increasing repetitions provides more instances to learn, it also makes the training more taxing. To

1087 maximize efficiency, training may ideally be provided in multiple sessions to avoid decrease in

1088 attention. Spacing the training also has the added benefit of promoting long-term behavior

1089 change (Bakkour et al., 2018), which is an important goal for behavior change interventions.

1090 Second, the effect of GNG was examined for choices between healthy and unhealthy

1091 foods. To date, preference change as a result of go/no-go responses has mostly been examined

1092 for choices between items from the same category (Bakkour et al., 2016; Salomon et al., 2018;

1093 Schonberg et al., 2014; Veling, Chen, et al., 2017; Zoltak et al., 2018). The current work thus

1094 extends the effects of GNG to cross-category choices. However, the task employed here cannot

1095 be used in applied settings directly, as it contains healthy and unhealthy items on both go and no-

1096 go trials. As the result on the unhealthy-trained condition shows, this may inadvertently create

1097 preferences for unhealthy items to which people respond. When used as an intervention, the

1098 training should contain only healthy items on go trials, and only unhealthy items on no-go trials

1099 (Lawrence, Sullivan, et al., 2015). This modification may also promote learning on a category

1100 level (e.g., healthy food = go, unhealthy food = no-go) rather than on an item level, which may

1101 make the effect more generalizable. Generalization of the effect from trained to untrained items

1102 is important for the application of GNG, and needs to be further pursued.

\section{Limitations and Future Directions}

1104 Due to the lack of untrained items, it is unclear whether preference for go items over no-

1105 go items reflects an effect of the go items, an effect of the no-go items, or a combination of both. 
1106 This difficulty in the interpretation of the results applies not only to the current research, but also

1107 to all previous work on CAT, where the same choice task has been used. Future work may pit

1108 untrained items with both go and no-go items in the choice task, to directly test whether the

1109 effects induced by CAT and GNG are different in nature.

1110 Slow choices seem resistant to the influences of GNG. Interestingly, previous work on

1111 CAT has similarly shown that the effect of CAT on preferences is also constrained to situations

1112 where people choose under time pressure (Veling, Chen, et al., 2017). These findings thus raise

1113 the question of whether slow choices can be trained at all. As mentioned earlier, in addition to

1114 GNG and CAT, the stop-signal task (SST) has also been used to change behavior, although the

1115 results seem mixed (e.g., Houben, 2011; Schonberg et al., 2014). The effect of SST on health

1116 behavior change is also smaller than that of GNG in recent meta-analyses (Allom et al., 2016;

1117 Jones et al., 2016). Nevertheless, SST is often proposed to strengthen top-down inhibitory

1118 control capacity for effortful inhibition of impulses triggered by objects (Houben, 2011). SST

1119 may have a stronger influence on choices when there is sufficient time for the top-down

1120 suppression of impulses, but this possibility remains to be tested.

1121 Lastly, it is unclear whether the observed results can generalize to other samples, such as

1122 overweight or obese individuals. Previous work has shown that repeated GNG with unhealthy

1123 food items facilitated weight loss in an overweight community sample (Lawrence, Sullivan, et al.,

1124 2015), suggesting that the effects are not confined to undergraduate students with healthy body

1125 weight. Furthermore, a recent study directly compared an undergraduate student sample to a

1126 clinical sample of morbidly obese individuals, and found that GNG changes food evaluation in

1127 both samples in similar ways (Chen et al., 2018b). However, important differences were also

1128 identified. Student participants learned the stimulus-response contingencies better than the 
1129 clinical sample, and the learning of stimulus-response contingencies predicted the effect of GNG

1130 on evaluation. In light of these findings, the generalizability of the current findings to different

1131 samples and contexts may depend on how well individuals can learn from training, which also

1132 needs to be further explored.

\section{Conclusion}

1134 To summarize, GNG reliably changed people's preference when they made choices with

1135 time limit, but not with unlimited time. This effect was still observed one week after training.

1136 Furthermore, item reward value did not moderate the effect. Overall, these findings are in line

1137 with the idea that preferences are dynamically constructed in choice situations (Slovic, 1995).

1138 The construction of preferences is influenced by recent learning histories (such as whether one

1139 has responded to certain items or not), especially when preferences need to be expressed quickly.

1140 By showing the reliability, generalizability and boundary condition of preference change induced

1141 by mere action versus inaction, the current work provides more insight into the underlying

1142 mechanism of the effect, how trainings that manipulate responding versus not responding to

1143 objects may be used in applied settings, and raise new questions on what people learn from

1144 repeated stimulus-response pairings and how these learned content impact preferences.

1145 


\section{Footnotes}

11471 - Experiments 1 and 2 were conducted simultaneously, by assigning participants to one of the

1148 two conditions in a counterbalanced order. For the sake of consistency with other experiments

1149 reported in the current paper, we refer to them as two separate experiments. The preregistration

1150 for Experiments 1 and 2 contained some inconsistency. In the introduction of the preregistration,

1151 we discussed previous work showing that the training effect is more pronounced for items that

1152 are perceived to be more appetitive (in line with the BSI theory discussed in the main text). In

1153 the Hypotheses section of the preregistration, we accordingly predicted an effect on choices for

1154 the high value pairs in the slow choice condition (i.e., Experiment 1), but not for the low value

1155 pairs. For the fast choice condition (i.e., Experiment 2), we did not have directional hypotheses.

1156 However, regarding the effect of GNG on item value as measured by the auction task, we

1157 predicted an effect for both Experiment 1 and 2, but failed to mention that this effect was

1158 predicted only for high-value items. Furthermore, in the Analysis Plan part of the preregistration

1159 we failed to mention these predictions, and instead described data analyses in an exploratory

1160 manner. In the main text, we present directional hypotheses in line with the ones outlined in the

1161 Hypotheses part (plus that the effect on the auction task was only expected for high-value items),

1162 to stay close to the BSI theory that we subscribed to while conducting the first two experiments.

1163 Note that these predictions are actually not in line with the results we observed. Furthermore, the

1164 preregistration failed to mention that in the choice task with time limit (i.e., Experiment 2), when

1165 participants failed to choose within 1.5 seconds, the image would be replaced by a prompt saying

1166 "Choose Faster!" for 500ms. This prompt was actually used when conducting the experiments.

1167 The method section in the main text now correctly mentions the use of this prompt when time

1168 limit was used in the choice task. 
11692 - The number of participants recruited exceeded the planned sample size by one in Experiment

11701,2 , and 7. This is because participants registered for the experiments via an online participation

1171 system, and despite the experimenters' close monitoring, the number of sign-ups exceeded the

1172 planned sample size before the sign-up portal could be closed. In the interest of retaining all data,

1173 we decided to not discard the data from the extra one participant for these three experiments. An

1174 overview of planned sample size and exclusion of participants for all experiments can be found

1175 in Table S1 in the Supplemental Materials.

1176 3- Note that we preregistered repeated-measures logistic regression as the planned analysis,

1177 while in power analysis we used paired-samples $t$ test as the planned analysis when calculating

1178 power. This inconsistency was resolved from Experiment 3 on. See Footnote 4 for a power

1179 simulation based on repeated-measures logistic regression.

11804 - For the power simulation, we first defined a beta distribution with shape parameters 3 and 2

1181 as the population distribution of the underlying probability of choosing go over no-go items. The

1182 mean of this beta distribution is $60 \%$, and the SD is $20 \%$, which is comparable to what we

1183 observed in the fast choice condition in Experiment 1. Furthermore, this population distribution

1184 corresponds to Cohen's $d$ of 0.5 , which is also close to previous meta-analyzed effect size

1185 (Allom et al., 2016). After defining the population distribution, we simulated 10000 experiments:

1186 for each experiment, we randomly sampled 60 data points from the population distribution, and

1187 these data points corresponded to the underlying probabilities of choosing go items for 60

1188 subjects. For each subject, we further generated 64 trials with the Bernoulli distribution $(0=$ no-

1189 go, 1 = go; each subject's probability of choosing go items was used as P(1)). Repeated-

1190 measures logistic regression was conducted on each simulated experiment. Overall, in about 90\% 
1191 of the 10000 experiments, the intercepts were significantly higher than 0 at the .05 level. In other

1192 words, the current setup has about $90 \%$ power to detect the effect.

11935 - Note that in Experiments 3 to 7, we removed the exclusion criterion that participants who was

1194 not between 18 and 26 years old would be excluded, and instead used age between 18 and 35 as

1195 an eligibility criterion when recruiting participants. The exclusion criterion on age was initially

1196 used in Experiment 1 and 2 to ensure homogenous samples for each experiment, which would

1197 then enable the comparison of results across experiments. We used a slightly larger age range to

1198 facilitate the recruitment of participants for later experiments, while still making the samples

1199 homogenous and similar. Furthermore, we also removed the exclusion criterion that participants

1200 who bid less than 25 cents on more than 40 snack items would be excluded (except in

1201 Experiment 7, where an auction task was again used to assess value of food items). This

1202 exclusion criterion is more relevant when an auction task is used, as participants may underbid in

1203 order to keep the monetary reward. This strategy is no longer applicable when they are asked to

1204 indicate how much they would like to consume different food items, as was the case in the

1205 remaining experiments, except Experiment 7. 


\section{References}

1208 Allom, V., Mullan, B., \& Hagger, M. (2016). Does inhibitory control training improve health behaviour? A meta-analysis. Health Psychology Review, 10, 168-186. http://doi.org/10.1080/17437199.2015.1051078

Bakkour, A., Botvinik-Nezer, R., Cohen, N., Hover, A. M., Poldrack, R. A., \& Schonberg, T. (2018). Spacing of Cue-approach Training Leads to Better Maintenance of Behavioral Change. Preprint, 1-39.

1214 Bakkour, A., Leuker, C., Hover, A. M., Giles, N., Poldrack, R. A., \& Schonberg, T. (2016). Mechanisms of choice behavior shift using cue-approach training. Frontiers in Psychology, 7, 1-12. http://doi.org/10.3389/fpsyg.2016.00421

1217 Bates, D. M., Maechler, M., Bolker, B., \& Walker, S. (2015). Fitting linear mixed-effects models using lme4. Journal of Statistical Software, 67, 1-48. http://doi.org/10.1177/009286150103500418

Berkman, E. T., Hutcherson, C. A., Livingston, J. L., Kahn, L. E., \& Inzlicht, M. (2017). SelfControl as Value-Based Choice. Current Directions in Psychological Science, 26(5), 422428. http://doi.org/10.1177/0963721417704394

Best, M., Lawrence, N. S., Logan, G. D., Mclaren, I. P. L., Best, M., Lawrence, N. S., ... Verbruggen, F. (2016). Should I stop or should I go? The role of associations and expectancies. Journal of Experimental Psychology: Human Perception and Performance, 42, 115-137. http://doi.org/http://dx.doi.org/10.1037/xhp0000116

Bowley, C., Faricy, C., Hegarty, B., Johnstone, S. J., Smith, J. L., Kelly, P. J., \& Rushby, J. A. (2013). The effects of inhibitory control training on alcohol consumption, implicit alcoholrelated cognitions and brain electrical activity. International Journal of Psychophysiology, 89(3), 342-348. http://doi.org/10.1016/j.ijpsycho.2013.04.011

Breslin, P. A. S. (2013). An evolutionary perspective on food and human taste. Current Biology, 23(9), R409-R418. http://doi.org/10.1016/j.cub.2013.04.010

1233 Chen, Z., Veling, H., Dijksterhuis, A., \& Holland, R. W. (2016). How does not responding to 1234 appetitive stimuli cause devaluation : Evaluative conditioning or response inhibition?

1235 Journal of Experimental Psychology: General, 145, 1687-1701. 
http://doi.org/10.1037/xge0000236

Chen, Z., Veling, H., Dijksterhuis, A., \& Holland, R. W. (2018a). Do impulsive individuals

1238 benefit more from food go/no-go training? Testing the role of inhibition capacity in the nogo devaluation effect. Appetite, 124, 99-110. http://doi.org/10.1016/j.appet.2017.04.024

Chen, Z., Veling, H., de Vries, S. P., Bijvank, B. O., Janssen, I., Dijksterhuis, A., \& Holland, R. Normal-Weight Individuals. Journal of Consulting and Clinical Psychology, 86, 980-990. http://doi.org/10.1037/ccp0000320

1244 Cunningham, W. A., \& Zelazo, P. D. (2007). Attitudes and evaluations: a social cognitive 1245 neuroscience perspective. Trends in Cognitive Sciences, 11(3), 97-104.

1246 http://doi.org/10.1016/j.tics.2006.12.005

1247 Cunningham, W. A., Zelazo, P. D., Packer, D. J., \& Van Bavel, J. J. (2007). The Iterative

1248 Reprocessing Model: A Multilevel Framework for Attitudes and Evaluation. Social

1249 Cognition, 25(5), 736-760. http://doi.org/10.1521/soco.2007.25.5.736

1250 Dayan, P., \& Niv, Y. (2008). Reinforcement learning: The Good, The Bad and The Ugly.

1251 Current Opinion in Neurobiology, 18(2), 185-196.

1252 http://doi.org/10.1016/j.conb.2008.08.003

1253 Doallo, S., Raymond, J. E., Shapiro, K. L., Kiss, M., Eimer, M., \& Nobre, A. C. (2012).

1254 Response inhibition results in the emotional devaluation of faces : neural correlates as 1255 revealed by fMRI. http://doi.org/10.1093/scan/nsr031

1256 Doya, K. (2008). Modulators of decision making. Nature Neuroscience, 11(4), 410-416.

1257 http://doi.org/10.1038/nn2077

1258 Dreisbach, G., \& Fischer, R. (2015). Conflicts as Aversive Signals for Control Adaptation.

1259 Current Directions in Psychological Science, 24(4), 255-260.

$1260 \quad$ http://doi.org/10.1177/0963721415569569

1261 Faul, F., Erdfelder, E., Lang, A.-G., \& Buchner, A. (2007). G*Power 3: A flexible statistical

1262 power analysis program for the social, behavioral, and biomedical sciences. Behavior

1263 Research Methods, 39, 175-191. http://doi.org/10.3758/BF03193146

1264 Fazio, R. H. (1990). Multiple Processes by Which Attitudes Guide Behavior: The MODE Model 1265 as an Integrative Framework. Advances in Experimental Social Psychology, 23, 75-109.

1266 Folkvord, F., Veling, H., \& Hoeken, H. (2016). Targeting implicit approach reactions to snack 
food in children: Effects on intake. Health Psychology, 35, 919-922. http://doi.org/10.1037/hea0000365

Forstmann, B. U., Ratcliff, R., \& Wagenmakers, E.-J. (2016). Sequential Sampling Models in Cognitive Neuroscience: Advantages, Applications, and Extensions. Annual Review of Psychology, 67, 641-66. http://doi.org/10.1146/annurev-psych-122414-033645

Friese, M., Hofmann, W., \& Wänke, M. (2008). When impulses take over: Moderated predictive validity of explicit and implicit attitude measures in predicting food choice and consumption behaviour. British Journal of Social Psychology, 47(3), 397-419. http://doi.org/10.1348/014466607X241540

Frischen, A., Ferrey, A. E., Burt, D. H. R., Pistchik, M., \& Fenske, M. J. (2012). The affective consequences of cognitive inhibition: Devaluation or neutralization? Journal of Experimental Psychology: Human Perception and Performance, 38(1), 169-179. http://doi.org/10.1037/a0025981

Fritz, J., \& Dreisbach, G. (2013). Conflicts as aversive signals: Conflict priming increases negative judgments for neutral stimuli. Cognitive, Affective and Behavioral Neuroscience, 13(2), 311-317. http://doi.org/10.3758/s13415-012-0147-1

Gollwitzer, P. M. (1999). Implementation intentions: Strong effects of simple plans. American Psychologist, 54(7), 493-503. http://doi.org/10.1037/0003-066X.54.7.493

Greenwald, A. G., McGhee, D. E., \& Schwartz, J. L. (1998). Measuring individual differences in implicit cognition: the implicit association test. Journal of Personality and Social Psychology, 74(6), 1464-80. http://doi.org/10.1037/0022-3514.74.6.1464

Guitart-Masip, M., Duzel, E., Dolan, R., \& Dayan, P. (2014). Action versus valence in decision making. Trends in Cognitive Sciences, 18, 194-202. http://doi.org/10.1016/j.tics.2014.01.003

Houben, K. (2011). Overcoming the urge to splurge: Influencing eating behavior by manipulating inhibitory control. Journal of Behavior Therapy and Experimental Psychiatry, 42, 384-388. http://doi.org/10.1016/j.jbtep.2011.02.008 stop responding to alcohol cues reduces alcohol intake via reduced affective associations rather than increased response inhibition. Addiction, 107, 1280-1287. http://doi.org/10.1111/j.1360-0443.2012.03827.x 
1298 Houben, K., \& Jansen, A. (2011). Training inhibitory control. A recipe for resisting sweet temptations. Appetite, 56, 345-349. http://doi.org/10.1016/j.appet.2010.12.017

Houben, K., \& Jansen, A. (2015). Chocolate equals stop. Chocolate-specific inhibition training reduces chocolate intake and go associations with chocolate. Appetite, 87, 318-323. http://doi.org/10.1016/j.appet.2015.01.005

Jones, A., Di Lemma, L. C. G., Robinson, E., Christiansen, P., Nolan, S., Tudur-Smith, C., \& Field, M. (2016). Inhibitory control training for appetitive behaviour change: A meta-

Jones, A., \& Field, M. (2013). The effects of cue-specific inhibition training on alcohol analytic investigation of mechanisms of action and moderators of effectiveness. Appetite,

Krajbich, I., Armel, C., \& Rangel, A. (2010). Visual fixations and the computation and

Lawrence, N. S., Verbruggen, F., Morrison, S., Adams, R. C., \& Chambers, C. D. (2015).

Leys, C., Ley, C., Klein, O., Bernard, P., \& Licata, L. (2013). Detecting outliers: Do not use standard deviation around the mean, use absolute deviation around the median. Journal of Experimental Social Psychology, 49(4), 764-766. http://doi.org/10.1016/j.jesp.2013.03.013 $492-527$. 
http://doi.org/10.1002/bs.3830090304

Peirce, J. W. (2007). PsychoPy-Psychophysics software in Python. Journal of Neuroscience Methods, 162(1-2), 8-13. http://doi.org/10.1016/j.jneumeth.2006.11.017

Porter, L., Bailey-Jones, C., Priudokaite, G., Allen, S., Wood, K., Stiles, K., ... Lawrence, N. S. (2018). From cookies to carrots; the effect of inhibitory control training on children's snack selections. Appetite, 124, 111-123. http://doi.org/10.1016/j.appet.2017.05.010

R Core Team. (2017). R Development Core Team. R: A Language and Environment for Statistical Computing. http://doi.org/http://www.R-project.org

Rangel, A., Camerer, C., \& Montague, P. R. (2008). A framework for studying the neurobiology

Salomon, T., Botvinik-Nezer, R., Gutentag, T., Gera, R., Iwanir, R., Tamir, M., \& Schonberg, T.

Schonberg, T., Bakkour, A., Hover, A. M., Mumford, J. A., Nagar, L., Perez, J., \& Poldrack, R. (2018). The Cue-Approach Task as a General Mechanism for Long-Term Non-Reinforced Behavioral Change. Scientific Reports, 8(1), 1-13. http://doi.org/10.1038/s41598-01821774-3 A. (2014). Changing value through cued approach: an automatic mechanism of behavior change. Nature Neuroscience, 17, 625-630. http://doi.org/10.1038/nn.3673

Seibt, B., Häfner, M., \& Deutsch, R. (2007). Prepared to eat: How immediate affective and motivational responses to food cues are influenced by food deprivation. European Journal of Social Psychology, 37(2), 359-379. http://doi.org/10.1002/ejsp.365

Serfas, B. G., Florack, A., Büttner, O. B., \& Voegeding, T. (2017a). What does it take for sour grapes to remain sour? Persistent effects of behavioral inhibition in go/no-go tasks on the evaluation of appetitive stimuli. Motivation Science, 3(1), 1-18. http://doi.org/10.1037/mot0000051

Serfas, B. G., Florack, A., Büttner, O. B., \& Voegeding, T. (2017b). What does it take for sour grapes to remain sour? Persistent effects of behavioral inhibition in go/no-go tasks on the evaluation of appetitive stimuli. Motivation Science, 3(1), 1-18. http://doi.org/10.1037/mot0000051

Slovic, P. (1995). The Construction of Preference. American Psychologist, 50(5), 364-371. http://doi.org/10.1037/0003-066X.50.5.364 
1360 Stice, E., Yokum, S., Veling, H., Kemps, E., \& Lawrence, N. S. (2017). Pilot test of a novel food response and attention training treatment for obesity: Brain imaging data suggest actions shape valuation. Behaviour Research and Therapy, 94, 60-70. http://doi.org/10.1016/j.brat.2017.04.007

Strack, F., \& Deutsch, R. (2004). Reflective and impulsive determinants of social behavior. Personality and Social Psychology Review : An Official Journal of the Society for Personality and Social Psychology, Inc, 8(3), 220-247. http://doi.org/10.1207/s15327957pspr0803_1

Sullivan, N., Hutcherson, C., Harris, A., \& Rangel, A. (2015). Dietary Self-Control Is Related to the Speed With Which Attributes of Healthfulness and Tastiness Are Processed. Psychological Science, 26(2), 122-134. http://doi.org/10.1177/0956797614559543

Sutton, R. S., \& Barto, A. G. (1998). Reinforcement Learning: An Introduction. MIT Press. http://doi.org/10.1109/TNN.1998.712192

Thorndike, E. L. (1911). Animal Intelligence: Experimental Studies. New York: Macmillan. http://doi.org/10.1097/00005053-191205000-00016

Tilman, D., \& Clark, M. (2014). Global diets link environmental sustainability and human health. Nature, 515(7528), 518-522. http://doi.org/10.1038/nature13959

Van Koningsbruggen, G. M., Veling, H., Stroebe, W., \& Aarts, H. (2014). Comparing two psychological interventions in reducing impulsive processes of eating behaviour: Effects on self-selected portion size. British Journal of Health Psychology, 19(4), 767-782. http://doi.org/10.1111/bjhp.12075

van Strien, T., Peter Herman, C., Engels, R. C. M. E., Larsen, J. K., \& van Leeuwe, J. F. J. (2007). Construct validation of the Restraint Scale in normal-weight and overweight females. Appetite, 49(1), 109-121. http://doi.org/10.1016/j.appet.2007.01.003

Veling, H., Aarts, H., \& Papies, E. K. (2011). Using stop signals to inhibit chronic dieters' responses toward palatable foods. Behaviour Research and Therapy, 49, 771-780. http://doi.org/10.1016/j.brat.2011.08.005

Veling, H., Aarts, H., \& Stroebe, W. (2013a). Stop signals decrease choices for palatable foods through decreased food evaluation. Frontiers in Psychology, 4, 1-7. http://doi.org/10.3389/fpsyg.2013.00875

Veling, H., Aarts, H., \& Stroebe, W. (2013b). Using stop signals to reduce impulsive choices for 
palatable unhealthy foods. British Journal of Health Psychology, 18, 354-368. http://doi.org/10.1111/j.2044-8287.2012.02092.x

Veling, H., Chen, Z., Tombrock, M. C., Verpaalen, I. A. M., Schmitz, L. I., Dijksterhuis, A., \& Holland, R. W. (2017). Training Impulsive Choices for Healthy and Sustainable Food. Journal of Experimental Psychology: Applied. http://doi.org/10.1037/xap0000112

Veling, H., Holland, R. W., \& van Knippenberg, A. (2008). When approach motivation and behavioral inhibition collide: Behavior regulation through stimulus devaluation. Journal of Experimental Social Psychology, 44(4), 1013-1019. http://doi.org/10.1016/j.jesp.2008.03.004

Veling, H., Lawrence, N. S., Chen, Z., van Koningsbruggen, G. M., \& Holland, R. W. (2017). What Is Trained During Food Go/No-Go Training? A Review Focusing on Mechanisms and a Research Agenda. Current Addiction Reports, 4, 35-41. http://doi.org/10.1007/s40429017-0131-5

Veling, H., van Koningsbruggen, G. M., Aarts, H., \& Stroebe, W. (2014). Targeting impulsive processes of eating behavior via the internet. Effects on body weight. Appetite, 78, 102-109. http://doi.org/10.1016/j.appet.2014.03.014

Verbruggen, F., Best, M., Bowditch, W. A., Stevens, T., \& McLaren, I. P. L. (2014). The inhibitory control reflex. Neuropsychologia, 65(312445), 263-278. http://doi.org/10.1016/j.neuropsychologia.2014.08.014

Verbruggen, F., \& Logan, G. D. (2008). Automatic and controlled response inhibition: associative learning in the go/no-go and stop-signal paradigms. Journal of Experimental Psychology. General, 137(4), 649-672. http://doi.org/10.1037/a0013170

Wessel, J. R., Doherty, J. P. O., Berkebile, M. M., Linderman, D., \& Aron, A. R. (2014). Stimulus devaluation induced by stopping action. Journal of Experimental Psychology: General, 143(6), 2316-2329. http://doi.org/http://dx.doi.org/10.1037/xge0000022

World Health Organisation. (2016). WHO | Obesity and overweight.

Zoltak, M. J., Veling, H., Chen, Z., \& Holland, R. W. (2018). Attention! Can choices for low value food over high value food be trained? Appetite, 124, 124-132. http://doi.org/10.1016/j.appet.2017.06.010 